University of South Florida

DIGITAL COMMONS

Digital Commons @ University of

@ UNIVERSITY OF SOUTH FLORIDA

South Florida

USF Tampa Graduate Theses and Dissertations

USF Graduate Theses and Dissertations

June 2017

\title{
Comparative Analysis of Tag Estimation Algorithms on RFID EPC \\ Gen-2 Performance
}

Arundhoti Ferdous

University of South Florida, arundhoti.ferdous@gmail.com

Follow this and additional works at: https://digitalcommons.usf.edu/etd

Part of the Electrical and Computer Engineering Commons

\section{Scholar Commons Citation}

Ferdous, Arundhoti, "Comparative Analysis of Tag Estimation Algorithms on RFID EPC Gen-2

Performance" (2017). USF Tampa Graduate Theses and Dissertations.

https://digitalcommons.usf.edu/etd/6837

This Thesis is brought to you for free and open access by the USF Graduate Theses and Dissertations at Digital Commons @ University of South Florida. It has been accepted for inclusion in USF Tampa Graduate Theses and Dissertations by an authorized administrator of Digital Commons @ University of South Florida. For more information, please contact digitalcommons@usf.edu. 


\title{
Comparative Analysis of Tag Estimation Algorithms on RFID EPC Gen-2 Performance
}

\author{
by
}

\begin{abstract}
Arundhoti Ferdous
A thesis submitted in partial fulfillment of the requirements for the degree of Master of Science in Electrical Engineering Department of Electrical Engineering College of Engineering University of South Florida
\end{abstract}

Major Professor: Ismail Uysal, Ph.D.

Nasir Ghani, Ph.D.

Selcuk Kose, Ph.D.

Date of Approval:

June 13, 2017

Keywords: Anti-collision, Passive RFID, Dynamic Frame Slotted ALOHA, Utilization Rate, Estimation Error

Copyright $\odot$ 2017, Arundhoti Ferdous 


\section{DEDICATION}

I dedicate my work to my parents, husband and my professor Dr. Ismail Uysal. 


\section{ACKNOWLEDGMENTS}

I would like to thank my supervisor Dr. Ismail Uysal from the bottom of my heart for his constant encouragement, supervision and support and for being patient with me from the very beginning of this research work. His guidance helped me to develop a good understanding of the subject.

I would also like to thank my parents - Mr. Kazi Abul Manjur and Mrs. Ferdous Ara Shanta and my family, without whom, I would not be here where I am today. Finally, I would like to thank my husband Ibrahim Azad whose constant support made this master's degree possible. 


\section{TABLE OF CONTENTS}

$\begin{array}{ll}\text { LIST OF TABLES } & \text { iii }\end{array}$

LIST OF FIGURES

ABSTRACT

CHAPTER 1: INTRODUCTION

1.1 Introduction to RFID 1

1.2 Basic Building Blocks of an RFID System 3

1.2.1 RFID Tags 4

1.2.1.1 Active Tags 4

1.2.1.2 Semi-Passive Tags 4

1.2.1.3 Passive Tags 4

1.2.2 RFID Reader 5

1.2.3 Data Processing Subsystems 5

1.3 RFID Operating Frequencies $\quad 5$

1.3.1 Low Frequency (LF) 6

1.3.2 High Frequency (HF) 6

1.3.3 Ultra-High Frequency (UHF) 6

$\begin{array}{ll}\text { 1.3.4 Microwave } & 7\end{array}$

$\begin{array}{ll}\text { 1.3.5 Ultra-Wideband (UWB) } & 7\end{array}$

1.4 Communication Principle of RFID 7

1.4.1 Near Field System $\quad 8$

1.4.2 Far Field System $\quad 8$

1.5 Applications of RFID 9

1.6 Challenges 12

CHAPTER 2: REVIEW OF ANTI-COLLISION ALGORITHMS AND THE INDUSTRY STANDARD FOR PASSIVE RFID SYSTEMS 15

2.1 Brief Description of Different RFID Anti-Collision Protocols $\quad 15$

2.1.1 Multiple Access / Anti-Collision Protocols 16

2.2 Tree Based Protocols $\quad 18$

2.3 ALOHA Based Protocols $\quad 18$

2.3.1 Classification of ALOHA Based Protocols $\quad 19$

$\begin{array}{ll}\text { 2.3.1.1 Pure ALOHA (PA) } & 19\end{array}$

2.3.1.1.1 Pure ALOHA with Muting 20

2.3.1.1.2 Pure ALOHA with Slow Down 20

2.3.1.1.3 Pure ALOHA with Fast Mode 20 
2.3.1.1.4 Hybrids 20

2.3.1.2 Slotted ALOHA (SA) 21

2.3.1.2.1 Slotted ALOHA with Muting and Slowdown 21

2.3.1.2.2 Slotted ALOHA with Early End 21

2.3.1.2.3 Slotted ALOHA with Early End and Muting $\quad 21$

2.3.1.2.4 Slotted ALOHA with Slowdown and Early End $\quad 21$

2.3.1.3 Frame Slotted ALOHA (FSA) 22

2.3.1.3.1 Basic Frame Slotted ALOHA (BFSA) 22

2.3.1.3.2 Dynamic Frame Slotted ALOHA (DFSA) 22

2.3.1.3.3 Enhanced Dynamic Frame Slotted ALOHA (EDFSA) 23

2.4 RFID Anti Collision Standards for ALOHA Based Systems 24

2.5 EPCglobal Class 1 Generation 2 Standard Protocol 24

CHAPTER 3: SELECTED TAG ESTIMATION ALGORTIHMS AND MAXIMUM

SYSTEM EFFICIENCY FOR DYNAMIC FRAME SLOTTED ALOHA 28

3.1 Factors Considered for Choosing the Algorithms 28

3.2 Selected Tag Estimation Algorithms 29

3.2.1 Two Conditional Tag Estimation Method 29

$\begin{array}{ll}3.2 .2 \text { DFSA-I } & 30\end{array}$

3.2.3 DFSA-II 30

3.3 Maximum System Efficiency in Dynamic Frame Slotted ALOHA 31

CHAPTER 4: EXPERIMENTAL DESIGN AND EVALUATION OF THE ALGORITHMS

W.R.T. EPC GEN-2 32

4.1 Experimental Design $\quad 32$

4.2 Parameters for Performance Evaluation $\quad 33$

4.2.1 Efficiency 33

4.2.2 Utilization Rate $\quad 34$

4.2.3 Estimation Error $\quad 34$

4.3 Tools and Simulation $\quad 34$

4.4 Performance Evaluation $\quad 35$

4.4.1 Efficiency vs Number of Tags 35

4.4.2 Effects of Utilization Rate and Estimation Error on Efficiency $\quad 42$

4.4.2.1 Utilization Rate vs Efficiency 42

4.4.2.2 Estimation Error vs Efficiency 51

CHAPTER 5: CONCLUSION $\quad 56$

$\begin{array}{lr}\text { REFERENCES } & 58\end{array}$ 


\section{LIST OF TABLES}

Table 2.1 Brief summary of the tree based protocols

Table 2.2 Brief comparison between tree and ALOHA based protocols

Table 2.3 Basic comparison of ALOHA based protocols

Table 2.4 ALOHA based anti-collision standards

Table 4.1 Performance comparison in terms of efficiency and standard deviation of the algorithms for $\mathrm{Q}=2, \mathrm{C}=0.2 \&$ Frame Size

Table 4.2 Performance comparison in terms of efficiency and standard deviation of the algorithms for $\mathrm{Q}=4, \mathrm{C}=0.2 \&$ Frame Size

Table 4.3 Performance comparison in terms of efficiency and standard deviation of the algorithms for $\mathrm{Q}=2, \mathrm{C}=0.2 \&$ (>Frame Size/2)

Table 4.4 Performance comparison in terms of efficiency and standard deviation of the algorithms for $\mathrm{Q}=4, \mathrm{C}=0.2 \&(>$ Frame Size/2)

Table 4.5 Performance comparison in terms of efficiency and standard deviation of the algorithms for $\mathrm{Q}=2, \mathrm{C}=0.2 \&$ No Gen-2

Table 4.6 Performance comparison in terms of efficiency and standard deviation of the algorithms for $\mathrm{Q}=4, \mathrm{C}=0.2 \&$ No Gen-2 


\section{LIST OF FIGURES}

Figure 1.1 Basic operation of RFID system 3

Figure 2.1 A common scenario of RFID system 15

Figure 2.2 Classification of anti-collision protocols 16

Figure 2.3 Flow diagram of updating Q parameter in EPC Gen-2 protocol 25

Figure 2.4 An illustrative example of the modification of Q parameter in EPC Gen-2 26

Figure 4.1 Flow diagram of Gen-2 incorporated with tag estimation algorithms 33

Figure 4.2 Efficiency comparison of the algorithms for Q = 2 \& Frame Size 36

Figure 4.3 Efficiency comparison of the algorithms for Q $=4$ \& Frame Size 37

Figure 4.4 Efficiency comparison of the algorithms for $Q=2 \&(>$ Frame Size/2) 37

Figure 4.5 Efficiency comparison of the algorithms for $Q=4 \&(>$ Frame Size/2) 38

Figure 4.6 Efficiency comparison of the algorithms for Q $=2 \&$ No Gen-2 38

Figure 4.7 Efficiency comparison of the algorithms for Q $=4$ \& No Gen-2 39

Figure 4.8 Histogram of utilization rate for DFSA-I, II \& P (Q = 2, C = $0.2 \&$ Frame Size $) 43$

Figure 4.9 Utilization rate vs efficiency for DFSA-I, II \& P (Q=2, C = $0.2 \&$ Frame Size $) 44$

Figure 4.10 Histogram of utilization rate for DFSA-I, II \& P (Q = 4, C = 0.2 \& Frame Size $) 45$

Figure 4.11 Utilization rate vs efficiency for DFSA-I, II \& P (Q=4, C = 0.2 \& Frame Size 46

Figure 4.12 Histogram of utilization rate for DFSA-I, II \& $\mathrm{P}(\mathrm{Q}=2, \mathrm{C}=0.2 \&>$ Frame Size/2)

Figure 4.13 Utilization rate vs efficiency for DFSA-I, II \& P (Q = 2, C = 0.2 \& > Frame Size/2) 
Figure 4.14 Histogram of utilization rate for DFSA-I, II \& $\mathrm{P}(\mathrm{Q}=4, \mathrm{C}=0.2 \&>$ Frame Size/2)

Figure 4.15 Utilization rate vs efficiency for DFSA-I, II \& $\mathrm{P}(\mathrm{Q}=4, \mathrm{C}=0.2 \&>$ Frame Size/2)

Figure 4.16 Estimation error vs efficiency for DFSA-I $(\mathrm{Q}=2 \& 4, \mathrm{C}=0.2 \&$ No Gen-2) 51

Figure 4.17 Histograms of estimation error for DFSA-I $(\mathrm{Q}=2 \& 4, \mathrm{C}=0.2 \&$ No Gen-2) 52

Figure 4.18 Estimation error vs efficiency for DFSA-II $(\mathrm{Q}=2 \& 4, \mathrm{C}=0.2 \&$ No Gen-2) 53

Figure 4.19 Histogram of estimation error for DFSA-II (Q=2 \& 4, C = $0.2 \&$ No Gen-2) 53

Figure 4.20 Histogram of estimation error for DFSA-P $(Q=4, C=0.2 \&$ No Gen-2) 54

Figure 4.21 Estimation error vs efficiency for $\operatorname{DFSA}-\mathrm{P}(\mathrm{Q}=4, \mathrm{C}=0.2 \&$ No Gen-2) 55 


\begin{abstract}
In a passive radio-frequency identification (RFID) system the reader communicates with the tags using the EPC Global UHF Class 1 Generation 2 (EPC Gen-2) protocol with dynamic framed slotted ALOHA. Due to the unique challenges presented by a low-power, random link, the channel efficiency of even the most modern passive RFID system is less than $40 \%$. Hence, a variety of methods have been proposed to estimate the number of tags in the environment and set the optimal frame size. Some of the algorithms in the literature even claim system efficiency beyond $90 \%$. However, these algorithms require fundamental changes to the underlying protocol framework which makes them ineligible to be used with the current hardware running on the EPC Gen-2 platform and this infrastructure change of the existing industry will cost billions of dollars. Though numerous types of tag estimation algorithms have been proposed in the literature, none had their performance analyzed thoroughly when incorporated with the industry standard EPC Gen-2. In this study, we focus on some of the algorithms which can be utilized on today's current hardware with minimal modifications. EPC Gen-2 already provides a dynamic platform in adjusting frame sizes based on subsequent knowledge of collision slots in a given frame. We choose some of the popular probabilistic tag estimation algorithms in the literature such as Dynamic Frame Slotted ALOHA (DFSA) - I, and DFSA - II, and rule based algorithms such as two conditional tag estimation (2CTE) method and incorporate them with EPC Gen-2 using different strategies to see if they can significantly improve channel efficiency and dynamicity. The results from each algorithm are also evaluated and compared with the performance of pure EPC
\end{abstract}


Gen-2. It is important to note that while integrating these algorithms with EPC Gen-2 to modify the frame size, the protocol is not altered in any substantial way. We also kept the maximum system efficiency for any MAC layer protocol using DFSA as the upper bound to have an impartial comparison between the algorithms. Finally, we present a novel and comprehensive analysis of the probabilistic tag estimation algorithms (DFSA-I \& DFSA-II) in terms of their statistically significant correlations between channel efficiency, algorithm estimation accuracy and algorithm utilization rate as the existing literature only look at channel efficiency with no auxiliary analysis. In this study, we use a scalable and flexible simulation framework and created a light-weight, verifiable Gen-2 simulation tool to measure these performance parameters as it is very difficult, if not impossible, to calculate system performance analytically. This framework can easily be used to test and compare more algorithms in the literature with Gen-2 and other DFSA based approaches. 


\section{CHAPTER 1: INTRODUCTION}

\subsection{Introduction to RFID}

In recent years, Radio frequency identification (RFID) technology has become one of the most commonly used technology in our daily life and is getting more prevalent in daily applications where automatic identification of objects is needed. RFID was first used in World War 2 by the Allied armed forces to discriminate friendly aircraft and tanks from enemy ones, called IFF (Identify Friend or Foe). Though it was first introduced decades ago, RFID technology has seen exponential gains in its application domains over the past decade or so. To cope with increasing logistical overheads, costs, product losses and overall inefficiency, both Department of Defense and Walmart authorized their respective delegates and suppliers to use RFID systems[1]. Now-a-days most of the supply chain giants and other dominant sectors use RFID. Some of the popular domains which use RFID technology are supply chain management, agriculture, military, healthcare, pharmaceuticals and retail.

Radio frequency identification (RFID) is an automatic identification process, where through the means of radio waves, required information is collected from a particular object which is to be identified without physical proximity or line of sight. Regardless of the type of the technology, i.e. the type and classification of the tags, readers, antennas and operating frequency, a typical RFID system consists of three main components: tags, reader and a data processing subsystem or server. It works by establishing a wireless link between the reader and the tag. A 
reader or interrogator identify objects that are labeled with RFID tags. A back end server or subsystem further processes and stores the information generated by the tag and the reader.

Although, RFID has similarities to the other automatic identification technologies such as barcodes, iris scanners, magnetic ink, touch memory etc., this technology has massive commercial prospective as it offers abundant benefits which are not feasible for more traditional identification technologies. Some of the advantages the technology possesses are [2]:

i) RFID tags have a larger read range than barcodes;

ii) In RFID systems, the tag's position is not a critical factor as in barcodes;

iii) Instead of being read one by one as in barcodes, multiple tags can be identified at the same time in this system, which makes it much more efficient.

iv) Unlike barcodes, RFID tags has a larger memory which enables them to store more data than just an ID.

v) RFID tags have read-write memory capability which allows the system to dynamically modify tag information as per the requirements of the application they are used for.

vi) RFID tags lessen the inventory control cost, save time and decrease errors as the identification process does not require human supervision.

vii) By combining sensors and other functionalities, RFID tags can perform auxiliary tasks like temperature monitoring, product quality calculation etc.

These features make RFID dominant when it comes to feasible commercial solutions. Having said that, it is critical that we understand RFID technology still faces a unique set of challenges [3]. It is also worth noting that different RFID technologies have different design requirements, protocols, performance specifications etc. to achieve the performance level they are known for. 
In this study, we are going to focus on the ultra-high frequency passive RFID systems which operate in $860 \mathrm{MHz}$ to $960 \mathrm{MHz}$ range. This particular type of RFID platform uses the industry standard EPC Gen-2 communication protocol [4] which is the prime focus of this study.

\subsection{Basic Building Blocks of an RFID System}

A typical RFID system involves a reading device or interrogator called a reader which communicates with one or more tags or transponders for identification. Upon receiving a signal from the reader, the tags get energized and send information as required by the reading device. A data processing server exists in the back end for receiving this information and for further processing and computing which facilitates both the tag and the reader. Figure 1.1 shows the basic operation of RFID [5, 6].



Figure 1.1: Basic operation of RFID system

In the following subsections, we will briefly discuss the basic building blocks of RFID. 


\subsubsection{RFID Tags}

Tags are one of the elementary units of RFID system. They are attached to the objects or products that are to be identified. A tag consists of an integrated circuit and a coupling element, i.e. antenna. Depending on the type of the tag, it may or may not have an on-board power source.

The type of tags defines the RFID variants. The choice of tags also outlines the area of application for which the RFID system is chosen for.

Based on their power usage, there are three types of RFID tags which are briefly presented in the following sections $[2,3,6,7]$.

\subsubsection{Active Tags}

Active tags are supported by ultra-high frequency and microwave systems. They are the most expensive ones due to their read and write capability, larger memory and on-board power source. The on-board power source accommodates the microchip and transceiver. Active tags can communicate independently and they do not have to rely on the reader's emitted power for communication. Active systems have a read range of more than 100 meters.

\subsubsection{Semi-Passive Tags}

Semi-passive tags are supported only by ultra-high frequency systems. Like active tags, semi passive tags also have an on-board power source but only used for powering the tag's microchip. They have to rely on the reader for data transmission. This type of tag is smaller compared to the active ones and has a shorter read range of 60 to 80 meters.

\subsubsection{Passive Tags}

This variant is supported by the low frequency, high frequency, ultra-high frequency and microwave systems. Passive tags have no internal power source. They have to rely solely on the reader's energy in order to energize and transmit. It has the smallest read range from 0.1 to 
typically 7 meters. Passive tags are the cheapest, thinnest and most flexible among the three which makes them very popular in the supply chain and retail industry.

\subsubsection{RFID Reader}

In an RFID system, a reader is one of the most important building blocks. A reader is basically a transceiver to interrogate the tags and read the information stored within. It has computational resources comparable to a computer. It generates and transmits electromagnetic wave through its antenna, internal or external to the reader and couples with the tags. It energizes and supplies power for data transmission to the tags if necessary. Readers can be classified in terms of mobility, i.e. stationary and mobile or handheld readers. They are generally connected to a computer or in the case of handheld readers a mobile computer can be built in the device providing a user interface to the user. For more sophisticated data processing, it sends the received information to the data processing subsystem or back end server. Hence the reader leaves most of the information and computational work for the connected back end server $[5,6,8]$.

\subsubsection{Data Processing Subsystems}

Irrespective of the type of the technology, i.e. the type of the readers and the tags, a back end database or server is used to facilitate the system operation. A backend system pools collected data together to enable big data analytics and drive business operations by interfacing with other resource management software [5].

\subsection{RFID Operating Frequencies}

One key parameter based on which RFID systems can be categorized further is the frequency range on which they operate. Each operating frequency range defines a certain operational characteristic as well as restrictions on different components of the RFID system. It basically outlines the desired application environment which they can be used for. In short, 
frequency is one of the most important factors one should consider when choosing an RFID system for a particular application for the most efficient use of the technology.

There are four major frequency ranges as briefly discussed below [3, 6, 8].

\subsubsection{Low Frequency (LF)}

In low frequency RFID systems, the tags operate in a frequency range which is less than $135 \mathrm{kHz}$. Most of the low frequency tags are passive tags which get their power from the reader. The read range is very small, typically up to 10 centimeters. Low frequency tags also have a data transfer rate of less than $10 \mathrm{kbits} / \mathrm{sec}$. Typical use of low frequency RFID tags can be found in animal tagging, access control, vehicle identification, container tracking in waste management etc.

\subsubsection{High Frequency (HF)}

High frequency RFID technology operates at $13.56 \mathrm{MHz}$ frequency which has a read range from 10 to 20 centimeters. The data transfer rate is less than $100 \mathrm{kbits} / \mathrm{sec}$. The tags are mostly passive in this frequency range.

Applications that entail moderate range are best fit for the high frequency tags. These tags are used mostly in access control, smart cards, item tagging, ticketing, document tracking, baggage control, laundries and libraries.

\subsubsection{Ultra-High Frequency (UHF)}

Ultra-high frequency RFID tags’ operating frequency range is from $860 \mathrm{MHz}-960 \mathrm{MHz}$. Although, these tags have an average read range of 5 to 6 meters, modern larger UHF tags in ideal conditions can operate up to $30+$ meter read range. The data transfer rate is $100 \mathrm{kbits} / \mathrm{sec}$. UHF RFID systems supports all three types of tags.

UHF RFID technology can be used in baggage handling, toll collection, supply chain management, pharmaceutical serialization, asset tracking, etc. 
This study focuses on UHF RFID systems, as the industry standard anti-collision protocol, Gen-2 is used for this frequency range.

\subsubsection{Microwave}

RFID systems with microwave tags also known as super-high frequency tags have an operating frequency range of $2.45 \mathrm{GHz}$ or $5.8 \mathrm{GHz}$. These tags have a very large read range and data transfer rate of 100 meters and less than $200 \mathrm{kbits} / \mathrm{sec}$ respectively. Microwave systems are more expensive than the three types mentioned earlier.

The application of microwave systems includes electronic toll collection, real time valuable goods tracking, production line tracking etc.

\subsubsection{Ultra-Wideband (UWB)}

This is relatively a recent technology compared to the previously mentioned ones. UWB tags have an operating frequency from $3.1 \mathrm{GHz}$ to $10.6 \mathrm{GHz}$. It offers a longer read range of 200 meters, lower cost and multi path signal resistance. It has great localization capabilities and can be used in hospitals for asset tracking as this technology can accommodate for both liquid and metal.

\subsection{Communication Principle of RFID}

In order to establish the primary wireless link between the tag and the reader, RFID uses radio waves. After being encoded and modulated by the user defined data, these signals are then transmitted, hence forming the basic functioning of RFID systems.

The two prime methods through which tag and reader communicate are magnetic and electromagnetic coupling. The difference between these two systems is based on the EM properties of RF antenna, mainly, the operating range. The operating field can be divided into the near field and the far field and they are briefly described in the following two sections $[3,6,8,9]$. 


\subsubsection{Near Field System}

The near field systems in RFID operate passively over the low frequency and high frequency spectrum. This is the system which uses magnetic field for coupling, i.e. the communication between the tag and the reader is obtained by magnetic induction. The term near field is defined as the region of one full wave length of the generated magnetic field by the reader's antenna. By producing a time varying magnetic field which results in an AC voltage, the reader induces a tag. As the antenna of both the reader and the tag aim to achieve the maximum transfer of energy, the inducing AC voltage is then rectified to a DC voltage in order to power up the tag's chip.

The reader modulates the magnetic field through amplitude modulation to transmit information to a tag. Whereas, a tag sends its ID to a reader by load modulation, i.e. by turning the load on and off. By recognizing the variations in amplitude and decoding the reader gets the ID sent by the tag.

Near field systems provides a robust link around dense RF media, excellent zone control, offers energy harvesting for passive high frequency and low frequency RFID systems. Having said that, it has many limitations as well such as the read range. Because the near field system is based on the magnetic induction process, as the frequency increases, the magnetic field's intensity decreases by $1 / r^{3}$, where $r$ is the distance between the tag and the reader, when measured along a perpendicular line from the reader antenna plane. The data rate and bandwidth are also limited which impacts the multi tag arbitration capability.

\subsubsection{Far Field System}

Far field systems are also known as the backscatter systems based on electromagnetic coupling. The system's operating region is ultra-high frequency and microwave range. As the 
name implies, the region defined by far field systems is greater than at least one full wavelength of the electromagnetic signal emitted by the reader's antenna. Both the reader and the tag use amplitude modulation technique and dipole antennas to transmit and receive signals. An AC voltage is generated at the reader's dipole antenna through a continuous electromagnetic wave, which creates a potential difference between the tag's dipole which in turn energize the tag's chip. In accordance with the data that is to be sent back to the reader the tag modulates the amplitude of the electromagnetic waves which are reflected by the tag's antenna, in other words "back scattering" the signal.

Far field systems have a much longer read range because of higher power. They also offer a higher multi tag arbitration capability as the bandwidth is larger. Though these systems have longer read ranges, they suffer two attenuation process during both transmission and reception. The first attenuation process occurs when the signal travels from the reader to the tag and the second process occurs when the signal is reflected back from the tag. Hence, the signal decreases by $1 / r^{4}$, where $r$ is the distance between the tag and the reader.

\subsection{Applications of RFID}

RFID technology has gained significant ground over the past decade because of a combination of technical and commercial factors. Because of the numerous advantages it possesses, RFID has abundant applications in real world applications like healthcare, agriculture, manufacturing, access control, supply chain, tracking, finance among many others. In this section, we will briefly discuss a few more popular and recent applications of RFID [2, 3, 9].

i) Logistic and supply chain visibility and management

This is one of the most popular and important application which is responsible for RFID technology to bloom. A supply chain involves a hectic environment of manufacturing, shipping, 
tracking, warehousing and retailing. In manufacturing sector from inventory management to resource tracking and quality control everything can be automated with RFID. Warehouse picking, receiving and shipping can be managed without human intervention. In the shipping sector, material processing, distribution and safety benefit from visibility provided by RFID. In today's world retailing is dominated by RFID, from inventory management to shelf stock checking, checking out management to point and time of purchase by the customer. RFID can track the shelf life of some perishable products to lessen wastage and improve quality. Supply chain giants like Walmart, Coca-Cola, Target and many more use RFID technology to track their assets every day. ii) Healthcare

RFID technology started growing in the health care sector over the last decade. It is being used for pharmaceuticals in keeping track of the drugs lifeline and preventing counterfeit drugs to ensure patient safety. It is also used for tracking hospital equipment and personnel. Patients' medical history, implant and prostheses are also tracked by this technology ensuring an authentic data base. Recently RFID is being used to take care of the elderly by tracking their movement with a device, either at healthcare facilities or their own homes.

iii) Access control

As everything is becoming more accessible in the wireless world it is important to maintain a certain level of security. RFID technology ensures access security from doors to parking lots, offices, clubs etc. by restricting the access only to authorized users.

iv) Agriculture

RFID tags are used in animal tracking for geolocation in a farm or by the owners. The tags are either implanted or clamped to their ears or body. This technology is also used in animal 
diagnostics and crop identification. They are used for early detection of viruses in livestock and bacterial, contaminant or pest exposures in crops.

v) Traffic, transportation, and ticketing

As one of the more successful and common applications of RFID systems, this area includes public transport tickets, congestion detection and management, intelligent traffic lights, finding thieves and offenders by automatic vehicle identification and tracking, public parking systems, toll collection, smart car keys and many more.

vi) Monitoring and tracking

Visibility and tracking with RFID are used in parcel mailbags, luggage handling, digital signature, laundry management, library inventory, IT assets and retailing. The supply chain giants keep track of their products by RFID systems which increase the store efficiency and make sure the products and goods are in stock.

vii) Human and object identification

This is the first application of RFID systems. During World War II, it was used to identify airplanes. Today, human identification is one of the major application areas of RFID. It includes digital ID, electronic passport, facility access etc. In all the cases, it increases efficiency and security.

viii) Finance

This field includes toll payment systems, smart cards, credit cards, bank note identification etc. RFID tags are used to process these remote transactions securely.

ix) Environment, fuel, chemicals

RFID system has its impact on environmental processes as well. It is used in waste haulage and recycling. After being attached to the waste, the tag will find its way to waste collection, 
treatment and disposal systems improving automation. It is also used to control the dispensing of fuel and chemicals automatically.

x) Government and military

Government assets, military logistics, IT assets, access control, and many important arear which are highly classified are controlled securely by RFID systems.

xi) Sports, games and entertainment industry

RFID systems are used in areas like tracking golf balls, game chips in the large casinos, sports event timing and ticketing etc. Disney theme parks also use RFID tags in form of hand bands to keep track of their clients and give them access to their parks and rides.

\subsection{Challenges}

Although we talked about RFID's enormous possibilities, the widespread use of this wireless system also brings technical, functional and security issues. As the number of tags sold in the market increases exponentially following key issues arise [2, 8, 9].

Standardization is one of the main issues as it leaves quite a bit of freedom in the choice of tags, communication protocol and format. It may create conflict as the companies need to agree on many factors like modulation type, data rate, encoding, frames, anti-collision algorithms etc.

Collision is another prime example of obstacles in RFID systems. As the number of tags in the read range increases and reader attempts to read multiple tags simultaneously, collision occurs which results in wastage of bandwidth, energy and time. Many anti-collision protocols show efficiency beyond $90 \%$ but it needs infrastructural change in the billion-dollar industry which is already in place. 
Cost is another issue when it comes to tags. As the required number of tags in fields like supply chain monitoring is only increasing the cost should go down even in the case of passive tags, to make it easier for the industry to adopt this technology.

It is difficult to decide on a certain frequency band because the choice depends on several key factors like the mode of transmission, the type and behavior of the tagged goods, environment in which the product is being read, international standards in frequency allocation etc.

Signal interference is another obstacle when a number of systems operate in the same environment. As this is a wireless system in dense environment where objects create signal scattering, refraction, and reflection communication becomes challenging.

Operating distance of the system is a crucial factor as well. The distance between the tag and reader antenna affects the power of the signal which decreases quadratically as the distance increases. This is an important issue in situations where the tag and/or the reader is not stationary.

For optimal system efficiency, antenna polarization and orientation also become an issue, especially in environments where the product orientation cannot be controlled.

$20 \%-30 \%$ of the tags manufactured are defective [2]. So, the faulty tags result in ineffective use of channel and bandwidth.

Faulty identifications by the reader are also crucial factors in RFID systems. It depends on many factors like adverse conditions of the environment, improper placement of the tagged products or the reader, accidental registration of data from unwanted tags which pass within the range of the reader unintentionally, reader malfunction etc.

As this is an extremely fast evolving industry, there are always new protocol standards which can perform faster and are more fault tolerant. This is another issue in the industry because the RFID technologies companies adopt quickly become outdated. 
Finally, security and privacy issues are always critical to RFID technology. Encryption must be ensured at all interfaces in order to ensure required security, to minimize unauthorized access especially for sensitive applications. 


\section{CHAPTER 2: REVIEW OF ANTI-COLLISION ALGORITHMS AND THE INDUSTRY STANDARD FOR PASSIVE RFID SYSTEMS}

\subsection{Brief Description of Different RFID Anti-Collision Protocols}

In a passive RFID system, the reader identifies the tags by initially broadcasting a query signal through its antenna(s). The tags respond to the reader's query command using backscatter modulation. If there is more than one tag transmitting signals at the same time, their packets collide resulting in failed transmission. This problem is known as tag collision, a major issue in RFID technology as it results in low identification accuracies, increased identification delays, wasted bandwidth and power [7]. In Figure 2.1, a common scenario of RFID system is depicted.

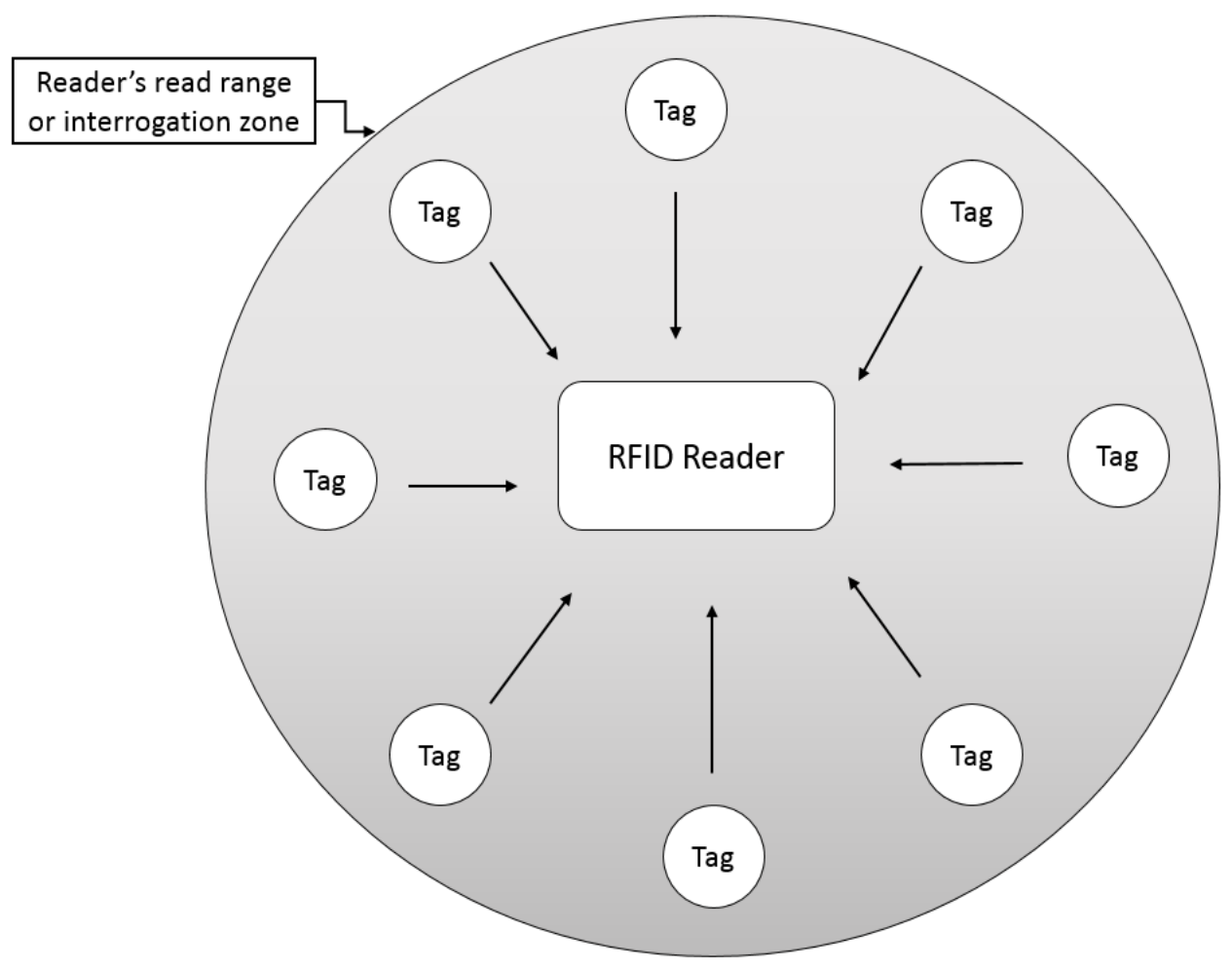

Figure 2.1: A common scenario of RFID system 
Due to random allocation of tags within the communication frame, the highest system efficiency is obtained when the current frame length is equal to the number of unidentified tags [5, 10]. Unfortunately, in a real-life application, unidentified tags are usually unknown to the reader, which makes it imperative to adopt effective anti-collision algorithms to enable a single reader to read multiple tags in the reader's field as effectively as possible. In this chapter, we go through the basic and major anti-collision protocols which make the basis of RFID technology $[3,6,11,12]$.

\subsubsection{Multiple Access / Anti-Collision Protocols}

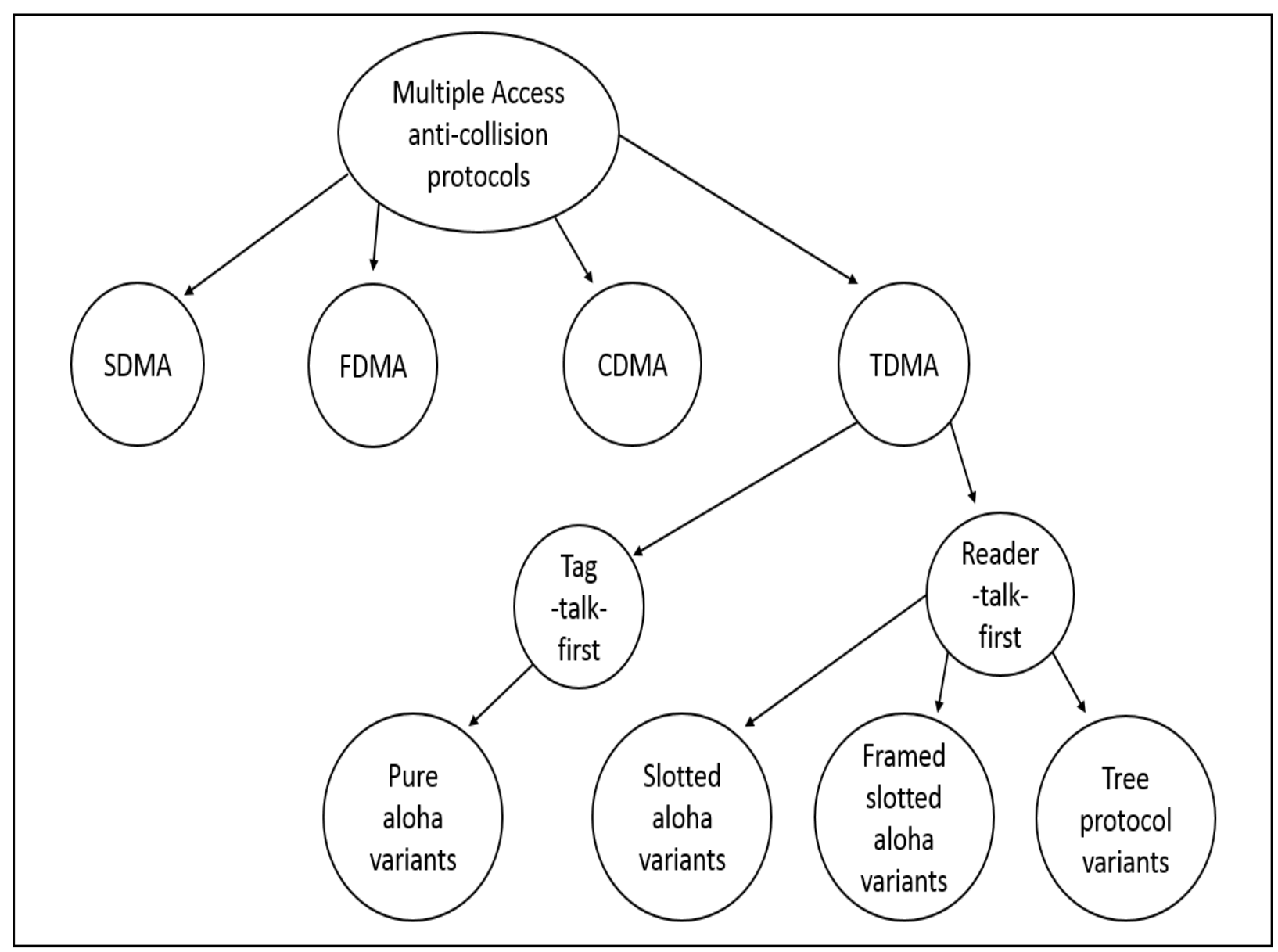

Figure 2.2: Classification of anti-collision protocols

As we can see from the Figure 2.2, the anti-collision protocols can be broadly classified into four multiple access protocols. 
In space division multiple access (SDMA), the channel is divided directionally by using either directional antennas or multiple readers for the identification process. For the spatial separation of the channel, this protocol requires complex antenna design.

In frequency division multiple access (FDMA), instead of dedicating the whole channel to a single communication link, the channel is divided into smaller bandwidths. Each frequency region is dedicated to a single tag until the identification is over. This mechanism requires complicated reader functionality to perform successful identifications.

In code division multiple access (CDMA), the tags are required to multiply their ID with a pseudo-random code before transmitting it to the reader. The reader then decodes the transmitted ID by matching them with a unique code. This system requires intricate functionality on both ends because of the computational work that needs to be done for the successful identification process.

Finally, in time division multiple access (TDMA), the channel bandwidth is divided into time slots which is used by the tags and the reader for the communication process. TDMA protocols forms the largest family of anti-collision protocols for passive RFID systems because of its ease of implementation and reduced complexity.

As seen from the figure TDMA systems can be further divided into reader driven systems also known as Reader-talk-first (RTF) and tag driven systems also known as Tag-talk-first (TTF). The former variant allows the reader to command first and the tags will remain silent until it is instructed otherwise by the reader. This system is synchronous. On the other hand, the latter works in an asynchronous manner, i.e. when a reader is present in the read zone of a tag, it declares itself. The TTF RFID systems are slow and more prone to collisions compared to the RTF systems. This is one of the reasons why RTF protocols are popular in most applications. 
TDMA protocols can be further classified into two broad categories, ALOHA and tree based protocols as probabilistic and deterministic approaches respectively.

\subsection{Tree Based Protocols}

Tree based protocols use a deterministic approach for slot allocation. Each tag is read with a unique ID by singling them out. For the tree based protocols to work, tags are required to have muting capability, i.e. the tags are silenced after identification process. A brief overview of existing tree based algorithms can be found in Table $2.1[3,6,13,14]$.

Table 2.1: Brief summary of the tree based protocols

\begin{tabular}{|c|c|c|c|c|}
\hline Criterion & $\begin{array}{c}\text { Query Tree } \\
\text { (QT) }\end{array}$ & $\begin{array}{c}\text { Tree Splitting } \\
\text { (TS) }\end{array}$ & $\begin{array}{c}\text { Binary Search } \\
\text { (BS) }\end{array}$ & $\begin{array}{c}\text { Bitwise } \\
\text { Arbitration } \\
\text { (BTA) }\end{array}$ \\
\hline Protocol feature & $\begin{array}{c}\text { The reader } \\
\text { and the tags with } \\
\text { prefix matching } \\
\text { the query } \\
\text { respond. }\end{array}$ & $\begin{array}{c}\text { The reader sends } \\
\text { a serial number } \\
\text { to the tags and } \\
\text { those with } \\
\text { tisjoint subsets, } \\
\text { the collisions are } \\
\text { resolved here. } \\
\text { values less than } \\
\text { or equal to the } \\
\text { serial number } \\
\text { reply. }\end{array}$ & $\begin{array}{c}\text { Every tag } \\
\text { responds in a bit } \\
\text { by manner }\end{array}$ \\
\hline RTF/TTF & \multicolumn{4}{|c|}{ Reader driven system } \\
\hline
\end{tabular}

Though tree based approach ensures higher throughput with a deterministic approach, it has complicated protocols and needs substantial memory overhead and complex hardware to implement. In the next section, we will briefly introduce ALOHA based protocols as they are the prime focus of this study.

\subsection{ALOHA Based Protocols}

Unlike the tree based protocols ALOHA uses a probabilistic approach for slot allocation. ALOHA based protocols are less complex, more robust and requires less bandwidth. Moreover, these protocols have dynamic adaptability to varying sizes of tag populations. In contrast to the tree based protocols, ALOHA based protocols have a limited number of reader to tag commands 
$[3,6,12,15]$. A brief comparison between the tree and ALOHA based protocols is presented in Table 2.2.

Table 2.2: Brief comparison between tree and ALOHA based protocols

\begin{tabular}{|c|c|c|}
\hline Criterion & Tree protocols & ALOHA protocols \\
\hline Protocol feature & $\begin{array}{c}\text { These protocols operate by } \\
\text { grouping responding tags into } \\
\text { subsets and then identifying } \\
\text { tags in each subset } \\
\text { sequentially. }\end{array}$ & $\begin{array}{c}\text { These protocols require tags } \\
\text { to respond randomly in an } \\
\text { asynchronous manner or in } \\
\text { synchronous time slots or } \\
\text { frames. }\end{array}$ \\
\hline Frequency & Mainly UHF and microwave & LF, HF and UHF \\
\hline Reader to tag commands & High & Low \\
\hline Efficiency versus tag density & Higher efficiency is & $\begin{array}{c}\text { Lesser efficiency in high tag } \\
\text { density }\end{array}$ \\
\hline Method & Deterministic & Probabilistic \\
\hline Optimum channel utilization & $43 \%$ & $\begin{array}{c}18.4 \% \text { (PA), 36.8\% (BFSA) } \\
\& 37 \% \text { (DFSA) }\end{array}$ \\
\hline
\end{tabular}

In this study, we'll concentrate on ALOHA protocols. In the next few sections we will briefly discuss different ALOHA based protocols which are classified in terms of randomization and frame allocation.

\subsubsection{Classification of ALOHA Based Protocols}

\subsubsection{Pure ALOHA (PA)}

In pure ALOHA, the reader sends out read requests which energize the tags. Each tag responds with ID randomly upon being energized. After that the tags wait for the reader's reply. It can be i) a positive acknowledgement (ACK) if the reader receives a tag's ID successfully or ii) a negative acknowledgement (NACK) if there is a complete or partial collision. For the latter case tags retransmit their ID after a random interval. However, this partial collision problem limits the throughput of the pure ALOHA based systems to $18 \%$. To improve the efficiency, this protocol has a few optimized versions which are discussed briefly in the following sections $[3,6,12,15]$. 


\subsection{Pure ALOHA with Muting}

In this type, when a tag is identified successfully by the reader, a 'mute' command is sent by the reader to that particular tag in order to make it stop responding to future read rounds. In this way, the tag load is lessened after each successful communication.

\subsection{Pure ALOHA with Slow Down}

Unlike the previous type, here, the reader sends a 'slowdown' command to a tag instead of the mute command after the tag has been successfully read. This slowdown command prolongs the back-off time of that particular tag which in turn reduces the collision probability.

\subsection{Pure ALOHA with Fast Mode}

In this variant of pure ALOHA, the reader sends out a silence command after it senses the start of a tag transmission. As a consequence, all the other tags go to the 'mute' mode. The tags unmute themselves either after getting an acknowledgement (ACK) command from the reader or after the expiration of their wait time.

\subsection{Hybrids}

By combining the variants mentioned above we can have two hybrid types: i) Pure ALOHA with fast mode and muting and ii) Pure ALOHA with fast mode and slowdown.

For the former type, the other tags are silenced using fast mode while a tag starts the transmission. After that particular tag is identified, the reader mutes the tag for the rest of the rounds ensuring a decreased amount of load.

In the latter variant, once a tag is identified using fast mode, it is slow downed, i.e.it is told by the reader to increase its back-off time, so that other tags can transmit with a lessened probability of collision. 


\subsubsection{Slotted ALOHA (SA)}

Unlike the pure ALOHA variant where tags respond with their ID on a continuous timeline, in the case of slotted ALOHA systems, tags transmit their ID in predefined synchronous time slots. Tags are required to respond only at the beginning of a slot so that the collision occurs only at the slot boundary. Because of this fitted synchronization there is no partial collision in the slotted ALOHA systems.

Slotted ALOHA has some variants as well which are briefly mentioned in the following sections $[3,6,12,15]$.

\subsection{Slotted ALOHA with Muting and Slowdown}

This type has the same operating principle as pure ALOHA with muting and slowdown but here the commands are utilized in a slotted manner.

\subsection{Slotted ALOHA with Early End}

In this variant, the reader is able to close the slot early if no transmission is detected at the starting of a slot. Two commands are used for this variant: i) Start-of-frame (SOF) to start a ready cycle, and ii) End-of-frame (EOF) to terminate an idle slot. These commands help to terminate an idle slot early which in turn helps with the read rate and channel efficiency.

\subsection{Slotted ALOHA with Early End and Muting}

This variant uses both early end and muting principles. A tag is muted by the reader after being identified and with the early end feature the reader can terminate a slot early by EOF command if it senses an idle slot.

\subsection{Slotted ALOHA with Slowdown and Early End}

This variant is similar to the previous one except instead of being muted an identified tag is slow downed by the reader. The reader also uses early end feature for this protocol. 


\subsubsection{Frame Slotted ALOHA (FSA)}

The main drawback in the previously mentioned protocols, i.e. pure and slotted ALOHA is that the tags respond randomly at least once in a read round which makes for a heavy tag load each time. This problem is overcome in frame slotted ALOHA protocol which restricts each tag to respond only once in each frame. Here in each reading cycle time is divided into frames and the frame is further divided into slots.

Based on how the reader implements and regulates the frame size, frame slotted ALOHA based systems are further divided into three main categories which are briefly discussed in the next few sections $[3,6,12,15]$.

\subsection{Basic Frame Slotted ALOHA (BFSA)}

In this variant, the word 'basic' stands for the fact that the frame length is not adjustable, and it remains constant throughout the reading process.

It has four customizations: i) BFSA-no-muting, ii) BFSA-with-muting, iii) BFSA-nomuting-early-end and iv) BFSA-with-muting-and-early-end, with "affixes" performing as explained in the section above.

\subsection{Dynamic Frame Slotted ALOHA (DFSA)}

The main downside of BFSA is that the frame size is fixed throughout the reading process which is a big problem when the number of tags significantly exceeds the frame size or vice versa.

DFSA overcomes this problem with adjustable frame size. Like BFSA, it also works in multiple read cycles and can employ early end feature. In order to adjust the frame size the reader needs to employ a tag estimation algorithm to estimate the number of tags and frame size. However, the limitation of this type is that the maximum frame size is restricted for stable operation. 


\subsection{Enhanced Dynamic Frame Slotted ALOHA (EDFSA)}

Enhanced dynamic frame slotted ALOHA protocol estimates the number of tags and matches it with number of slots in the current frame size to see if it would yield maximum system efficiency or not. If not, the tags are divided into $\mathrm{m}$ groups and the protocol calculates what should be the value of $\mathrm{m}$, i.e. how many number of groups are required. When the reader sends a tag respond in ordered groups.

Table 2.3: Basic comparison of ALOHA based protocols

\begin{tabular}{|c|c|c|c|c|c|}
\hline Criterion & $\begin{array}{c}\text { Pure } \\
\text { ALOHA } \\
\text { (PA) }\end{array}$ & $\begin{array}{c}\text { Slotted } \\
\text { ALOHA } \\
\text { (SA) }\end{array}$ & $\begin{array}{c}\text { Basic Framed } \\
\text { Slotted } \\
\text { ALOHA } \\
\text { (BFSA) }\end{array}$ & $\begin{array}{c}\text { Dynamic } \\
\text { Framed } \\
\text { Slotted } \\
\text { ALOHA } \\
\text { (DFSA) }\end{array}$ & $\begin{array}{c}\text { Enhanced } \\
\text { Dynamic } \\
\text { Framed } \\
\text { Slotted } \\
\text { ALOHA } \\
\text { (EDFSA) }\end{array}$ \\
\hline $\begin{array}{c}\text { Protocol } \\
\text { Feature }\end{array}$ & $\begin{array}{l}\text { Tag's ID is } \\
\text { transmitted at } \\
\text { random } \\
\text { times. If } \\
\text { collision } \\
\text { occurs it } \\
\text { retransmits } \\
\text { after a } \\
\text { random } \\
\text { delay. }\end{array}$ & $\begin{array}{l}\text { Tag's ID is } \\
\text { transmitted in } \\
\text { slots, if } \\
\text { collision } \\
\text { occurs it will } \\
\text { retransmit } \\
\text { after a random } \\
\text { number of } \\
\text { slots. }\end{array}$ & $\begin{array}{l}\text { A tag only has } \\
\text { one chance to } \\
\text { transmit in a } \\
\text { particular } \\
\text { frame and the } \\
\text { frame size is } \\
\text { fixed for the } \\
\text { whole process. }\end{array}$ & $\begin{array}{c}\text { A tag } \\
\text { transmits only } \\
\text { once in each } \\
\text { frame and the } \\
\text { frame size is } \\
\text { dynamic } \\
\text { based on the } \\
\text { tag density. }\end{array}$ & $\begin{array}{l}\text { If tag density } \\
\text { exceeds } \\
\text { maximum } \\
\text { frame size, } \\
\text { then the tags } \\
\text { are divided } \\
\text { into groups } \\
\text { and allowed } \\
\text { to transmit in } \\
\text { groups. }\end{array}$ \\
\hline $\begin{array}{c}\text { Disadvant } \\
\text { age }\end{array}$ & $\begin{array}{l}\text { Because of } \\
\text { partial } \\
\text { collision, as } \\
\text { the tag } \\
\text { density } \\
\text { increases, the } \\
\text { collision } \\
\text { increases } \\
\text { exponentially }\end{array}$ & $\begin{array}{l}\text { Poor handling } \\
\text { of high tag } \\
\text { density and } \\
\text { needs } \\
\text { synchronizati } \\
\text { on between } \\
\text { reader and } \\
\text { tags. }\end{array}$ & $\begin{array}{l}\text { Tags require } \\
\text { synchronizatio } \\
\text { n circuits. } \\
\text { Need to know } \\
\text { the current } \\
\text { frame size for } \\
\text { maximum } \\
\text { efficiency. }\end{array}$ & $\begin{array}{c}\text { Requires } \\
\text { sophisticated } \\
\text { readers which } \\
\text { monitors slots } \\
\text { with single } \\
\text { and no } \\
\text { responses } \\
\text { along with } \\
\text { collision. }\end{array}$ & $\begin{array}{l}\text { Needs a very } \\
\text { complicated } \\
\text { reading } \\
\text { device in } \\
\text { order to group } \\
\text { the tags. }\end{array}$ \\
\hline
\end{tabular}

In table 2.3 a brief summary and comparison of the ALOHA based protocols are given as described earlier in this chapter. 


\subsection{RFID Anti Collision Standards for ALOHA Based Systems}

RFID standards that use ALOHA based systems are listed in Table 2.4 below. These standards are managed and maintained by two main bodies: i) EPCglobal and ii) International Organization for Standardization (ISO). The former is responsible for developing standards which are targeted for international supply chain networks and the latter one is responsible for specifying air interface for tracking cattle, payment systems, smart cards and vicinity cards.

Table 2.4: ALOHA based anti-collision standards

\begin{tabular}{|c|c|c|}
\hline Standard & Frequency & Dedicated Protocol \\
\hline ISO 18000-3 "MODE 1" & HF & Pure ALOHA and DFSA \\
\hline ISO 18000-3 "MODE 2" & HF & $\begin{array}{c}\text { Combination of both TDMA and FDMA, uses } \\
\text { slotted ALOHA during transmission }\end{array}$ \\
\hline ISO 14443-3 Type-B & HF & DFSA \\
\hline ISO-18000-6A & UHF & FSA with muting and early-end \\
\hline EPCglobal Class 1 Gen 2 & UHF & Q protocol which uses DFSA \\
\hline EPCglobal Class 1 & HF & BFSA with early-end \\
\hline Philips I Code & HF & DFSA \\
\hline
\end{tabular}

Apart from these bodies we also have propriety standards an example of which is shown for Philips [3, 6].

In this study, we will concentrate on EPC Gen-2 protocol. This is the dominant industry standard for passive RFID systems which uses Q protocol with dynamic frame slotted ALOHA to solve the collision problem by adjusting the value of $\mathrm{Q}$ which in turn adjusts the frame size in each cycle. The next section is dedicated to this industry standard with a more detailed description of its working mechanism.

\subsection{EPCglobal Class 1 Generation 2 Standard Protocol}

EPC Gen-2 is the global industry standard for passive RFID systems operating in the frequency range of $860 \mathrm{MHz}$ to $960 \mathrm{MHz}$. This standard is built on the Q-protocol which uses 
DFSA $[4,5]$. The flow diagram indicating how the frame size is updated in this standard protocol is shown in Figure 2.3.

The protocol requires the tags to have a random number generator and a slot counter. As the reader sends a QUERY command to start the inventory cycle by setting the value of the main protocol parameter $Q$ for the tags, the tags must randomly select a number between 0 to $2^{Q}-1$ and store it in their slot counter. The value of Q is an integer which ranges from 0 to 15 and sets the frame size as $2^{Q}$.

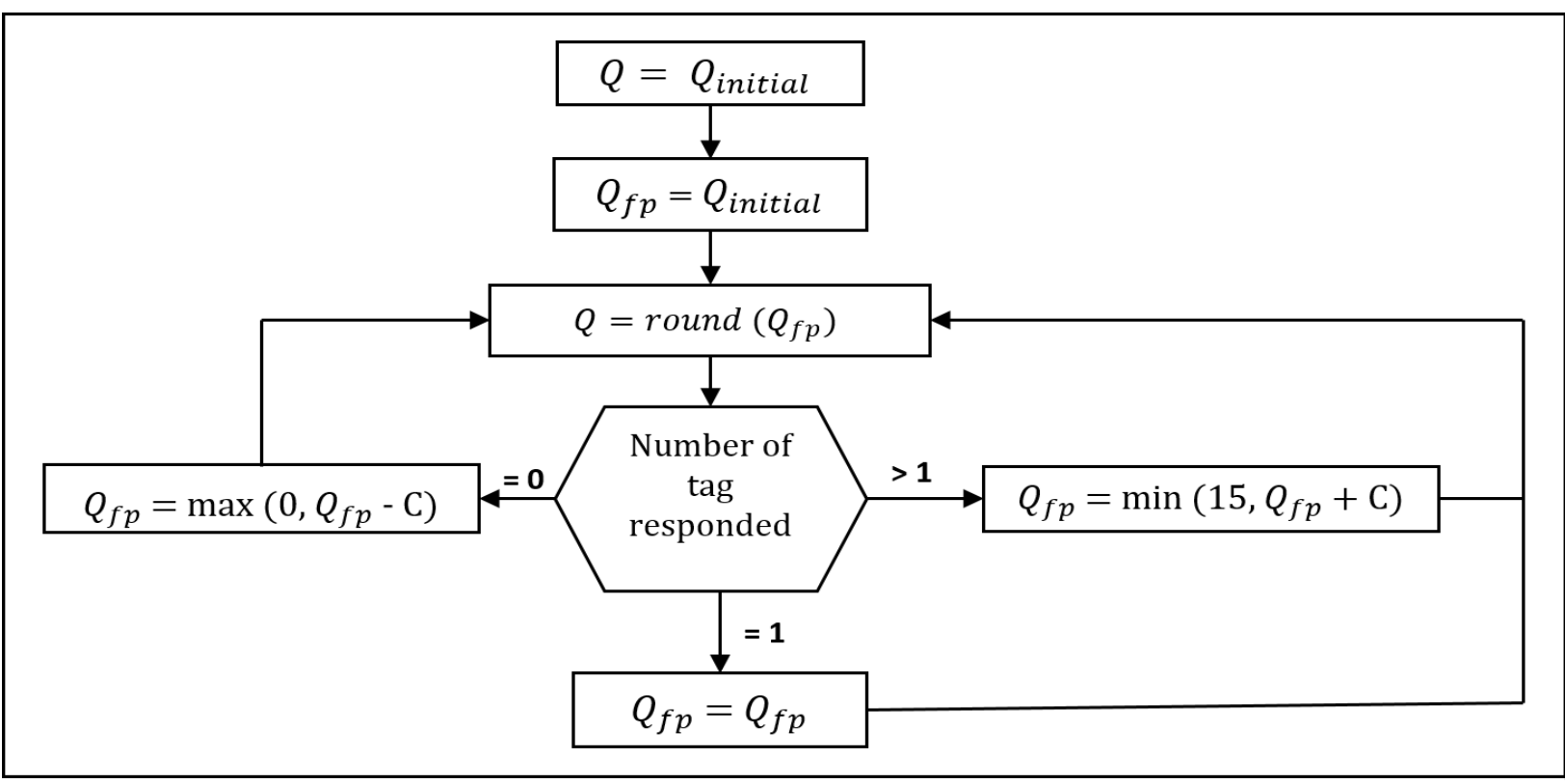

Figure 2.3: Flow diagram of updating Q parameter in EPC Gen-2 protocol

The random number picked by each tag represents the slot in the frame in which that particular tag can reply to the reader. The tag/s which picked random number 0 reply instantly by issuing a random 16-bit identification number, $\mathrm{RN}-16$, by using the random number generator. Other tags remain silent but reduce their slot counter by 1 and wait until their turn comes.

Three possibilities may arise after the tags communicate with the reader:

i) Idle slot: No tag responds to the reader within the specified time limit by the protocol; 
ii) Successful slot: Only one tag replying i.e. only one received signal which matches the slot number;

iii) Collision slot: More than one tag respond in the same slot.

\section{An Example of how Gen-2 works:}
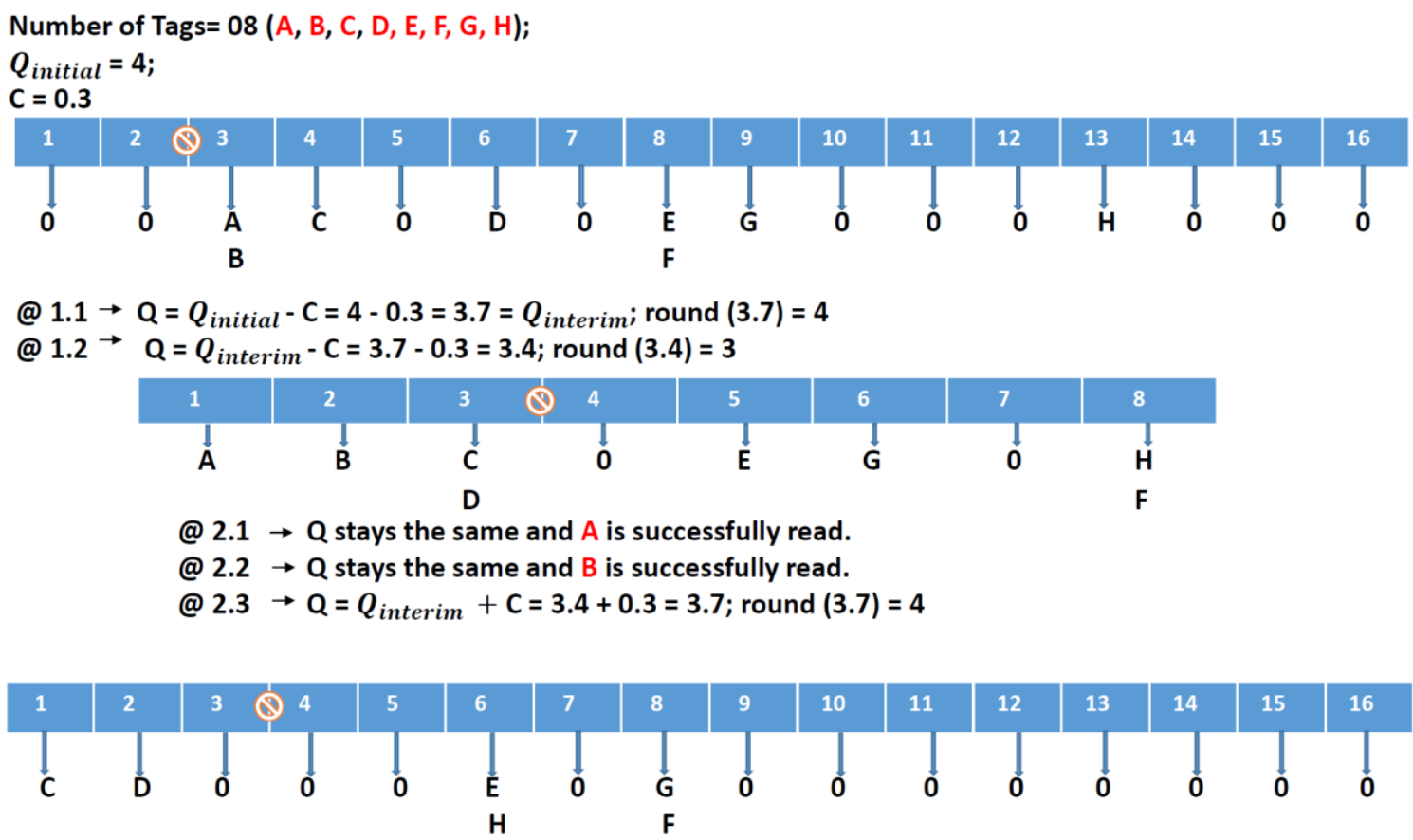

@ $3.1 \rightarrow Q$ stays the same and $C$ is successfully read.

@ $3.2 \rightarrow Q$ stays the same and $D$ is successfully read.

@ $3.3 \rightarrow Q=Q_{\text {interim }}-\mathrm{C}=3.7-0.3=3.4$; round $(3.4)=3$

\begin{tabular}{|c|c|c|c|c|c|c|c}
1 & 2 & 3 & 4 & 5 & 6 & 7 & 8 \\
\hline $\mathbf{E}$ & $\mathbf{F}$ & $\mathbf{H}$ & $\mathbf{G}$ & 0 & $\mathbf{H}$ & 0 & 0 \\
\hline
\end{tabular}

$@ 4.1 \rightarrow Q$ stays the same and $E$ is successfully read.

@ $4.2 \rightarrow Q$ stays the same and $F$ is successfully read.

@ $4.3 \rightarrow \mathrm{Q}=Q_{\text {interim }}-\mathrm{C}=3.4-0.3=3.1$; round $(3.1)=3$

@ $4.4 \rightarrow Q$ stays the same and $G$ is successfully read.

@ $4.5 \rightarrow \mathrm{Q}=Q_{\text {interim }}-\mathrm{C}=3.1-0.3=2.8$; round $(2.8)=3$

$@ 4.6 \rightarrow Q$ stays the same and $H$ is successfully read.

Figure 2.4: An illustrative example of the modification of Q parameter in EPC Gen-2

The protocol continues this procedure until all the tags in the read range are identified. The most important feature of this protocol is that it adjusts its frame size based on whether a slot is idle, successful or collision as shown in Figure. 2.3. The reader modifies the value of Q by using another protocol parameter $\mathrm{C}$ which varies from 0.1 to 0.5 which is typically preset depending on 
the tag density in the environment in which the reader is functioning. An illustrative scenario of how the frame size is updated in EPC Gen-2 protocol is shown below in Figure 2.4.

In Figure 2.4 we can see that the number of tags is 8 , initial value of $\mathrm{Q}$ is 4 and $\mathrm{C}$ is set to 0.3. In the first read cycle the frame breaks in the second slot and no tag is identified successfully. However, in the second round two tags are read successfully but the frame breaks after the third slot. Two more tags are identified in the third read cycle and the frame breaks again after the third slot. In the fourth cycle, the rest of the tags (four) are read successfully and the reading process is over. We can see that the frame size varies in each cycle which is determined by the Q protocol. In the following chapter, we will briefly introduce different types of tag estimation algorithms which will be incorporated with EPC Gen-2 in this study. 


\section{CHAPTER 3: SELECTED TAG ESTIMATION ALGORTIHMS AND MAXIMUM SYSTEM EFFICIENCY FOR DYNAMIC FRAME SLOTTED ALOHA}

\subsection{Factors Considered for Choosing the Algorithms}

A wide variety of tag estimation algorithms are reported in the literature, some of which claim efficiencies beyond $90 \%[16,17]$. However, these algorithms introduce fundamental changes to the underlying protocol framework [16-21] which makes them ineligible to be used with the existing hardware running on the EPC Gen-2 [4] platform.

In this study, we focus on algorithms which do not require substantial updates to the protocol and can be utilized on today's current hardware with minimal modifications. EPC Gen-2 is already dynamic in adjusting frame sizes based on a posterior knowledge of collusion slots in a given frame. For this work, we chose from popular tag estimation algorithms in the literature [2226] which are: DFSA-I, DFSA-II [27], 2CTE method [28] and incorporated them with EPC Gen2 to see if they can significantly improve its dynamic aspect and compare where they stand in terms of system efficiency with and without it. The performance of these tag estimation algorithms are also evaluated and compared with the performance of pure EPC Gen-2. It is important to note that while integrating these algorithms with EPC Gen-2 to modify the frame size, the protocol is not altered in any substantial way.

We also kept the maximum system efficiency for any MAC layer protocol using DFSA [5, 10] as the upper bound (which is called DFSA Perfect in this study) to have an impartial 
comparison between the algorithms. In the following sections, we will briefly introduce different types of tag estimation algorithms which will be incorporated with EPC Gen-2.

\subsection{Selected Tag Estimation Algorithms}

\subsubsection{Two Conditional Tag Estimation Method}

The 2CTE method claims that the best approach to estimate the number of unresolved tags would be to decrease the number of collision and idle slots. In 2CTE, the highest system efficiency in DFSA (when the frame size equals the number of unresolved tags in each cycle) $[5,10]$ is taken as a reference point in order to determine the optimal percentage of successful, collision and idle slots. The optimal average percentages for successful (OSS), collision (OCS) and idle (OFS) slots are calculated and given as $37 \%, 26 \%$ and $37 \%$ respectively. The actual percentage of successful (CSS), collision (CCS) and idle (CFS) slots are then measured to calculate the differences between the measured and optimal values and probabilistically estimate the number of actual tags. Two steps have been suggested here:

i) Decreasing collision slots:

- If CCS $>50 \%$ then the next frame size is increased faster.

- If $26 \%<\mathrm{CCS}<50 \%$ then to decrease the collision rate, the next frame size is increased.

ii) Decreasing free slots:

- If CCS $<26 \%$ \& CFS $<50 \%$ then the previous frame size is taken into account to decrease the current one.

- If CCS $<26 \%$ \& CFS $>50 \%$ then the frame size is decreased faster.

After each round of frame identification, the algorithm chooses the best condition to estimate the number of unresolved tags. The reader can refer to the original paper for details on the numerical estimation [28]. 


\subsubsection{DFSA-I}

In this algorithm [27], to estimate the number of tags $(n)$, the collision ratio $\left(C_{\text {ratio }}\right)$, i.e. the ratio of the number of the slots with collision to the frame size $(\mathrm{L})$ is defined by,

$$
C_{\text {ratio }}=1-\left(\frac{1}{L}\right)^{n}\left(1+\frac{n}{L-1}\right)
$$

After one complete round, the collision ratio can be measured. Since the frame size is already known, based on this information, one can estimate the number of tags by sweeping the variable n over a suitable range of values to find the one that best fits the calculated $C_{\text {ratio }}$ and the frame size [27]. This algorithm has some drawbacks not addressed in the original article, including the extreme boundary conditions such as when $C_{\text {ratio }}$ becomes 1 . In those instances, DFSA-1 provides inaccurate estimation of the number of tags and a variation of the algorithm, DFSA-II, is used to estimate the number of tags as described below.

\subsubsection{DFSA-II}

In this variation [27], to obtain the number of colliding tags in a slot, the collision rate $\left(C_{\text {rate }}\right)$ is defined as follows:

$$
C_{\text {rate }}=\frac{\text { Probability that there is a collision in a slot }}{1-\text { Probability that a tag transfers successfully }}
$$

From $[5,10]$, we know that the system reaches maximum throughput when optimal frame size $\left(L_{\text {optimal }}\right)$ is equal to the number of tags $(n)$. Hence, the optimal collision rate Crate for maximum throughput can subsequently be found as:

$$
C_{\text {rate }}=\lim _{n \rightarrow \infty} \frac{P_{\text {coll }}}{1-P_{\text {succ }}}=0.4180
$$

The number of tags $\left(C_{\text {tags }}\right)$ collided in a slot is then calculated by,

$$
C_{\text {tags }}=\frac{1}{C_{\text {rate }}}=2.3922
$$


Let $M_{\text {coll }}$ be the number of collided slots in a frame after a round. The estimated number of the tags $(n)$ is then calculated by,

$$
n=2.3922 * M_{\text {coll }}
$$

\subsection{Maximum System Efficiency in Dynamic Frame Slotted ALOHA}

For the evaluation of the tag estimation algorithms, i.e. how they perform with and without EPC Gen-2, we kept the maximum system efficiency for any MAC layer protocol using DFSA [10] as the upper bound which is named DFSA perfect (DFSA-P) here in this thesis. The expected maximum efficiency can be estimated from the following equation:

$$
E\left\{e f f_{\max }\right\}=n\left(\frac{1}{N}\right)\left(1-\frac{1}{N}\right)^{n-1}
$$

where, $\mathrm{N}$ is the frame size $\& \mathrm{n}$ is the responding tag population which indicates the optimal value for expected maximum efficiency and can be defined as follows:

$$
n=\left\lceil-\frac{1}{\ln \left(1-\frac{1}{N}\right)}\right\rceil
$$

Based on this calculation, the expected maximum efficiency is approximately $37 \%$ when the total number of tags in the read range is more than 50 [5]. In DFSA-P, we assume that the tag estimation algorithm works with a theoretical $100 \%$ accuracy, which means at the end of a frame one can know exactly how many unresolved tags remain in the read field. Similar to 2CTE, DFSAI and DFSA-II, we used this algorithm with and without EPC Gen-2, to compare how each tag estimation algorithm would have performed if their accuracies were closer to the highest theoretical accuracy compared to simply using pure EPC Gen-2 algorithm. 


\section{CHAPTER 4: EXPERIMENTAL DESIGN AND EVALUATION OF THE ALGORITHMS W.R.T. EPC GEN-2}

\subsection{Experimental Design}

In this study, we incorporate EPC Gen-2 [4, 5] with 2CTE [28], DFSA-I and DFSA-II [27] algorithms as described in the previous section to see if they can improve the efficiency of this standard protocol. As another baseline comparison, we include a perfect estimation algorithm (DFSA-P) which simply assumes the number of tags in the environment at every step of the simulation is known. EPC Gen-2 protocol adjusts its frame size by the parametric variable C depending on whether the slot is idle, successful or in collision [5]. Admittedly, this pattern of frame size update is dynamic by itself however no study has looked at how much more dynamic and efficient it can get when supported by different tag estimation algorithms. The EPC Gen-2 protocol is modified only when the frame size is updated. Otherwise, we kept the reader-tag communication method intact as per the standard protocol. The proposed experimental framework is shown in Figure 4.1.

Three scenarios are considered where the algorithms are used:

i) Only when there is no frame update until the very end of the frame, i.e., if the frame breaks early then the frame size is updated purely by EPC Gen-2.

ii) Only when the frame update occurs after mid-frame, if not then EPC Gen-2 takes over.

iii) In the absence of any EPC Gen-2 frame update mechanism, i.e. the algorithms are solely responsible for determining the next frame size. 


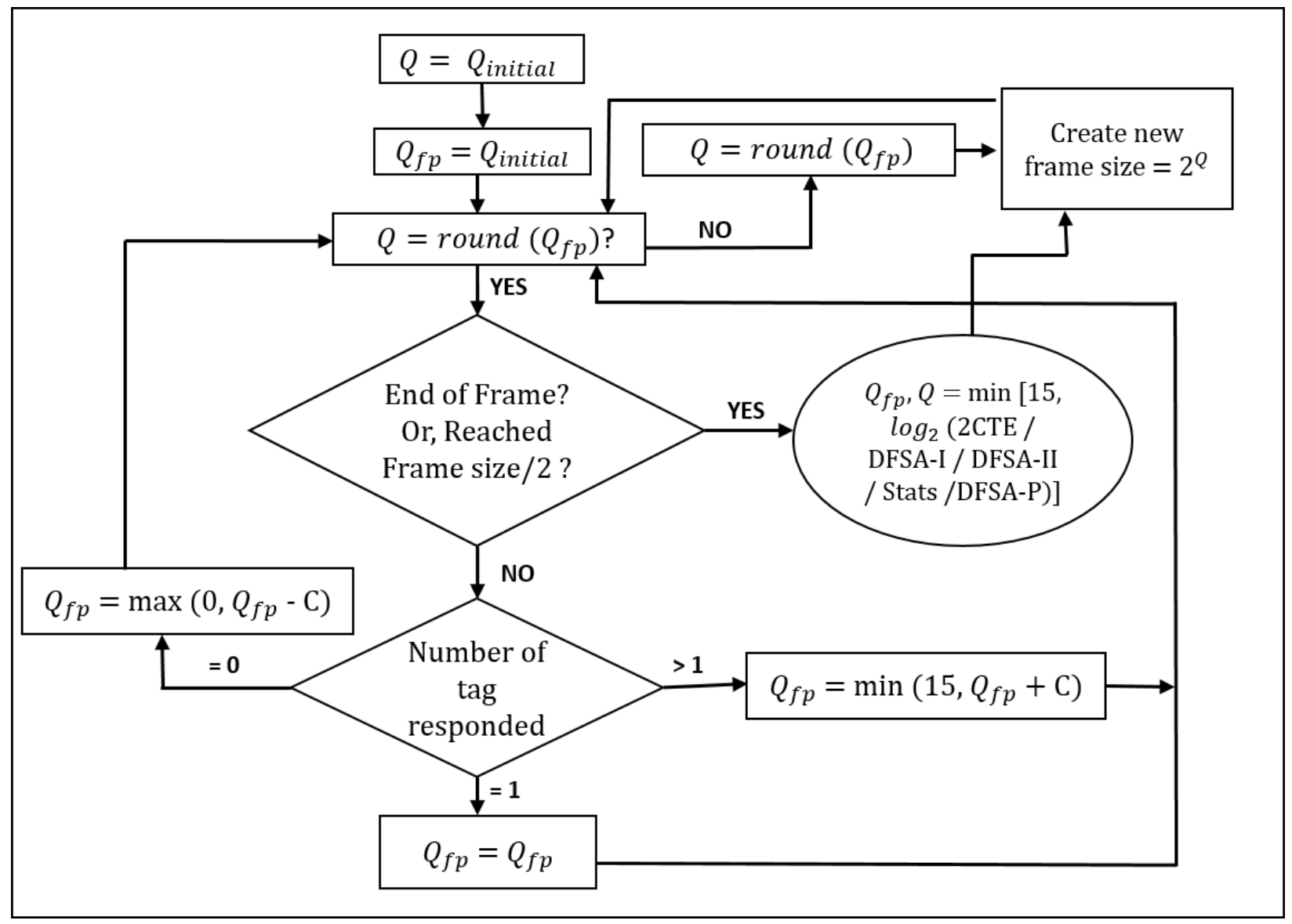

Figure 4.1: Flow diagram of Gen-2 incorporated with tag estimation algorithms

If EPC Gen-2 breaks the frame and either of these conditions are met, the new frame size is created with the modified $Q$ value and the size of the new frame is equal to the minimum of $2^{15}$ and $2^{Q}$ (where, $\mathrm{Q}$ is modified by the tested algorithm). Otherwise $\mathrm{Q}$ value is modified by EPC Gen-2, as usual. We can see in Figure4.1. DFSA-P (perfect) $[5,10]$ is included along with the other algorithms. This represents an upper bound in estimation accuracy.

\subsection{Parameters for Performance Evaluation}

\subsubsection{Efficiency}

We kept efficiency as the first parameter for comparing the effects of the algorithms which is defined as follows. 


$$
\operatorname{Eff}(\%)=\frac{N}{N_{T}} * 100 \%
$$

where, $N=$ number of tags in the read range $\& N_{T}=$ total number of slots used for the identification process of the entire tag population.

\subsubsection{Utilization Rate}

We evaluated the algorithms when they performed with and without EPC Gen-2. However, for the EPC Gen-2 case, we also kept track of how often the algorithms were used in resolving all the tags. Here the aim is to see the relationship between the efficiency and how often algorithms are utilized. We measured utilization rate for each algorithm by:

$$
\frac{\text { the number of times when the algorithm is used }}{\text { the total number of times when a new frame is created }}
$$

\subsubsection{Estimation Error}

Another parameter we chose for evaluating the tag estimation algorithm is the estimation error, i.e. how accurately the algorithms can estimate the number of tags in the read range. We wanted to see if the accuracy of the algorithms help boost the overall efficiency. The estimation error is calculated as follows:

$$
\frac{\text { actual number of tags - estimated number of tags }}{\text { actual number of tags }}
$$

\subsection{Tools and Simulation}

It's difficult to calculate such efficiency improvement analytically, so we designed our own simulation approach to measure these performance parameters. We started from [30], where a simulation tool for EPC gen-2 is developed. We eliminated all the auxiliary parameters from that algorithm and focused only on the system efficiency and a light-weight, verifiable simulation tool in MATLAB precisely designed and validated for this standard protocol. To evaluate the 
performance of the algorithms under different scenarios and with and without EPC Gen-2 we modified it and presented a scalable and flexible simulation framework. We did the modification at the frame size update stage based on the three scenarios as described earlier.

\subsection{Performance Evaluation}

As we discussed earlier, we evaluated the tag estimation algorithms based on three parameters: 1. Efficiency; 2. Utilization rate \& 3. Estimation error. For each performance assessing parameter we settled for literature standard choices of $\mathrm{C}=0.2$ and $\mathrm{Q}=2 \& 4$ for the standard protocol. The simulations were performed for $\mathrm{N}=10,100,300 \& 500$, where $\mathrm{N}$ is the number of tags to evaluate both low and high tag density environments. The number iteration for each simulation is subject to the statistical significance of performance evaluating parameters.

For efficiency, for $\mathrm{N}=10,100,300 \& 500$, the simulations were repeated for a minimum of 1000 times to achieve the highest statistical significance and consistent results.

As for the other two parameters, i.e. estimation error and utilization rate, we fixed the number of tags to be $100(\mathrm{~N}=100)$ for a more in-depth look. We also chose only DFSA-I and DFSA-II for this study because these algorithms show the highest compatibility w.r.t Gen-2 and DFSA-P. The other algorithm, i.e. 2CTE did not perform as well in terms of system efficiency. The simulations were repeated for 200,000 times in order to obtain enough data points for analyzing the statistical behavior of the algorithms.

\subsubsection{Efficiency vs Number of Tags}

We compared the performance of each algorithm in terms of efficiency with and without EPC Gen-2 to the following reference points.

i) Pure EPC Gen-2;

ii) EPC Gen-2 supported with DFSA-P i.e. the maximum theoretical estimation accuracy \& 
iii) Pure DFSA-P.

The efficiency comparisons of the algorithms with and without EPC Gen-2 under different scenarios alongside pure EPC Gen-2 and DFSA-P are summarized in the following figures. As discussed earlier, for comparing efficiencies we considered three scenarios:

i) When frame update occurs at the end of the frame;

ii) When frame update occurs after mid-frame;

iii) When there is no EPC Gen-2 frame update mechanism, i.e. the tag estimation algorithms perform on their own, at the end of each frame.

For all the scenarios and Q values of 2 and 4, we compared the efficiency of the algorithms for $\mathrm{N}=10,100,300 \& 500$.

The following two figures are for the first case, i.e. frame update occurs at the end of the frame for both $\mathrm{Q}$ values.

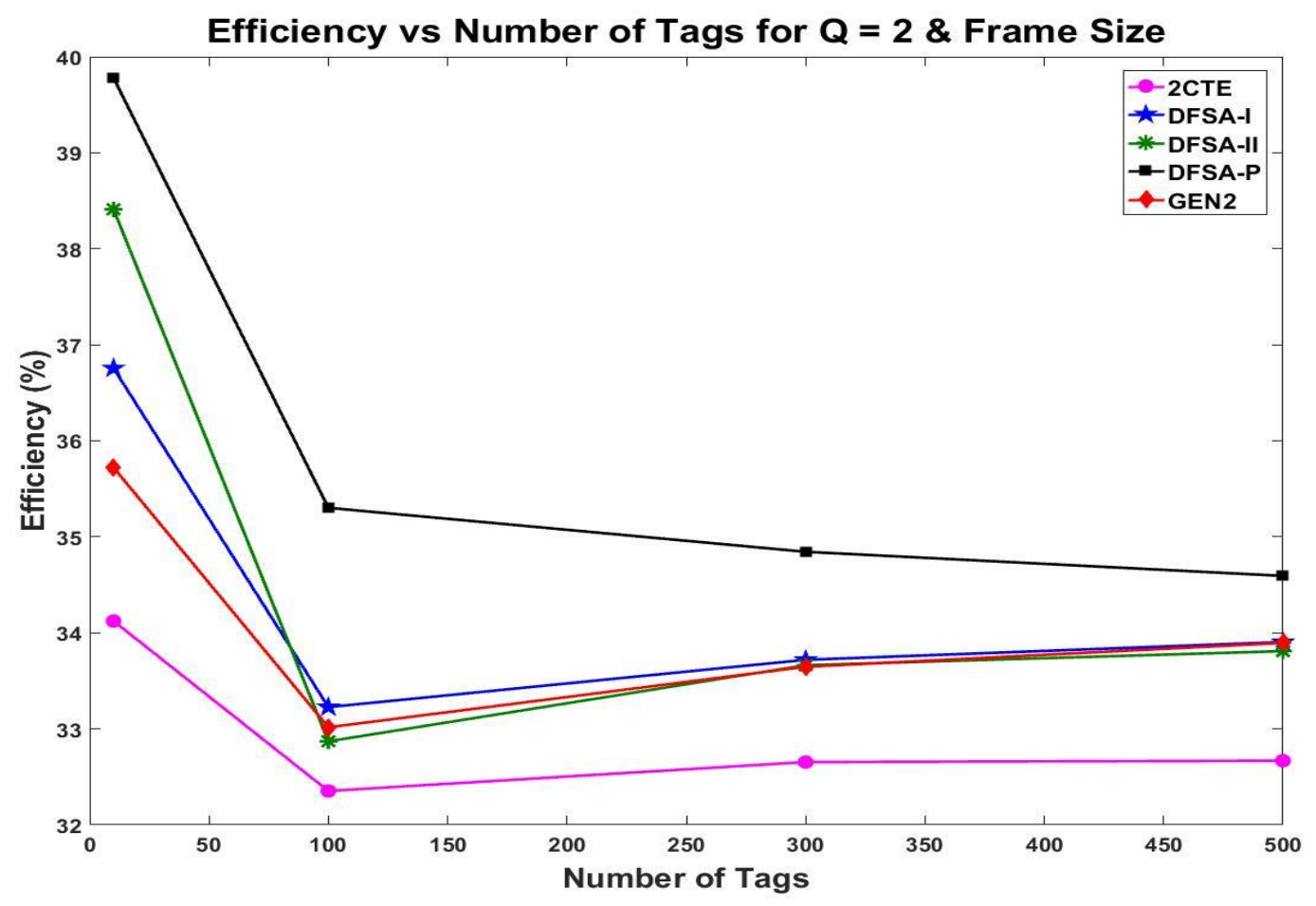

Figure 4.2: Efficiency comparison of the algorithms for $Q=2 \&$ Frame Size 




Figure 4.3: Efficiency comparison of the algorithms for $Q=4 \&$ Frame Size

We can see from Figure 4.2 and 4.3, at low tag densities (0-100) DFSA-II out performs other algorithms. However, as the number of tags increases all algorithms including Gen-2 performs quite similarly. It is noticeable, that for $\mathrm{Q}=4$, DFSA-II and perfect DFSA (DFSA-P) perform quite similarly. For both combinations, 2CTE method lags in terms of efficiency.

The following two figures are for the second case, i.e. when the frame update occurs after the mid-frame for $\mathrm{Q}=2$ and 4 .

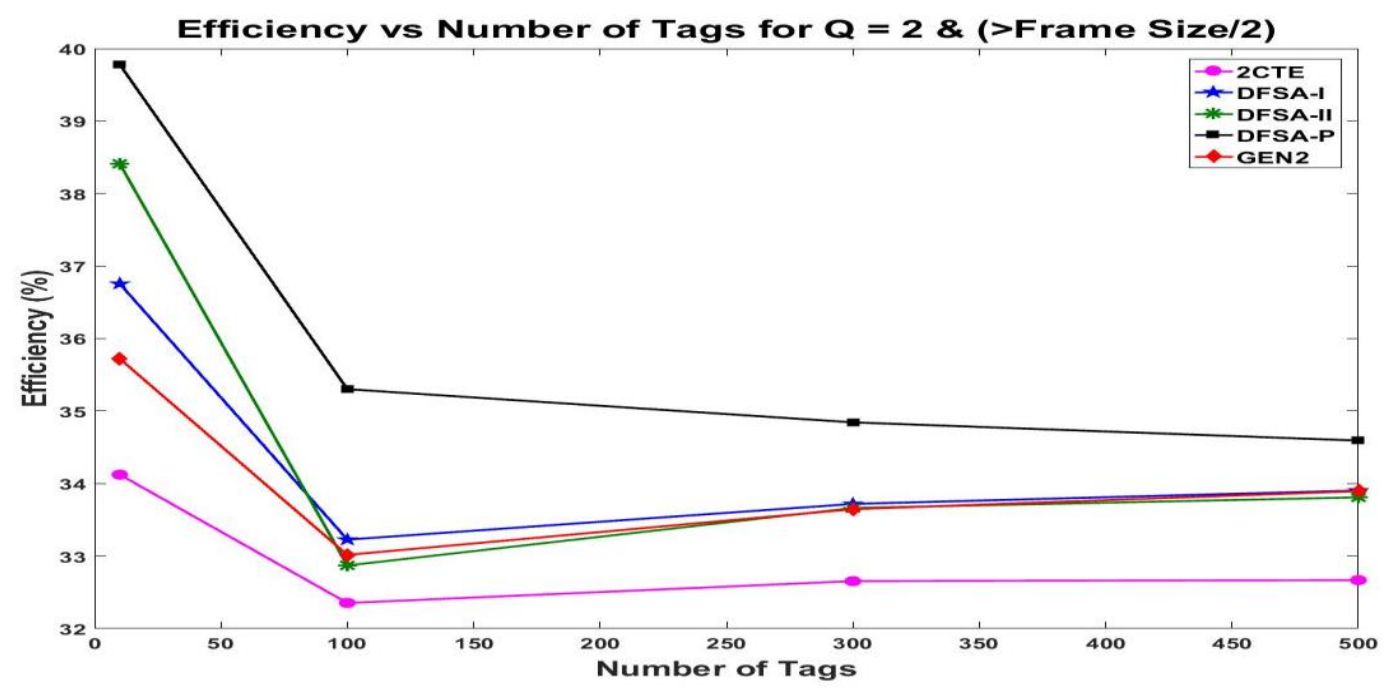

Figure 4.4: Efficiency comparison of the algorithms for $\mathrm{Q}=2 \&$ (>Frame Size/2) 


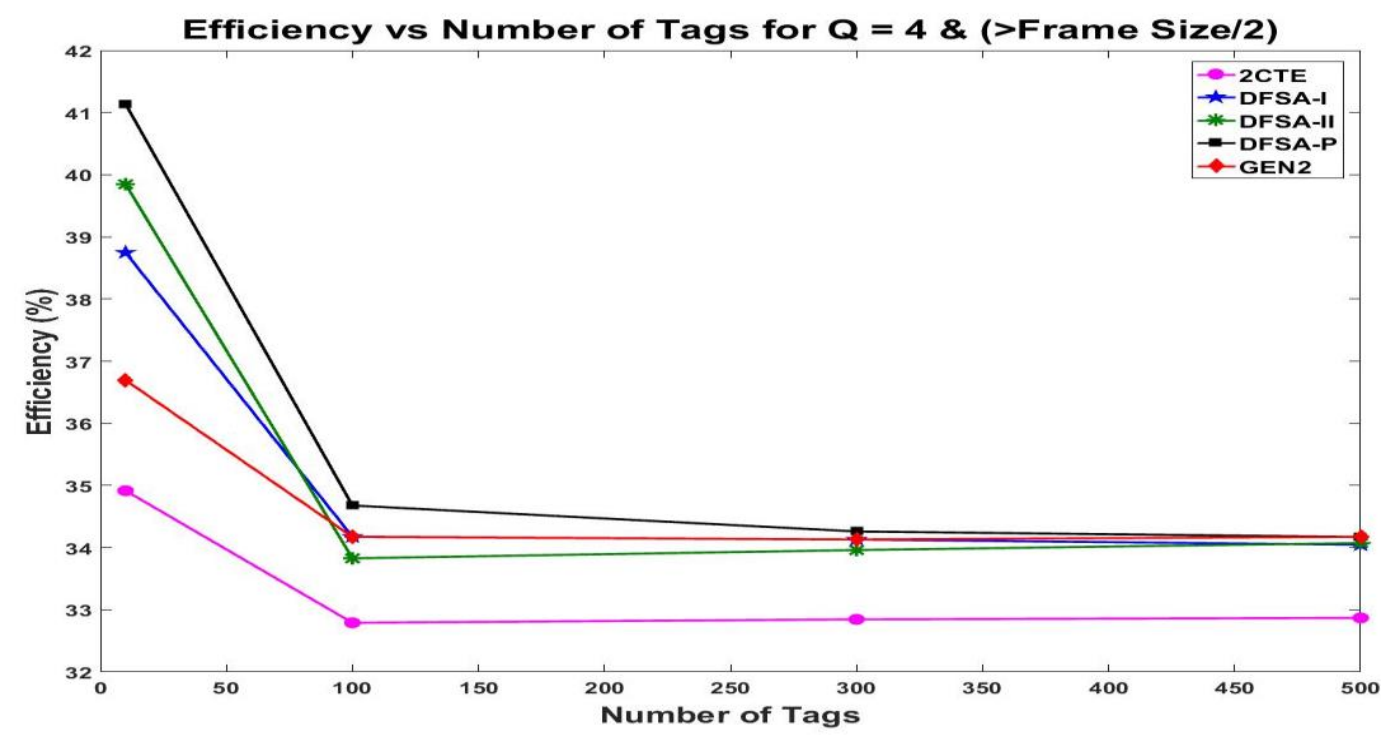

Figure 4.5: Efficiency comparison of the algorithms for $Q=4 \&$ (>Frame Size/2)

For this case, in both figures (4.4 and 4.5), we can see the same behavior as in the case shown in Figure 4.2 and 4.3.

The final two figures for efficiency comparison are for the third case, i.e. when there is no EPC Gen-2 frame update mechanism for both Q values.

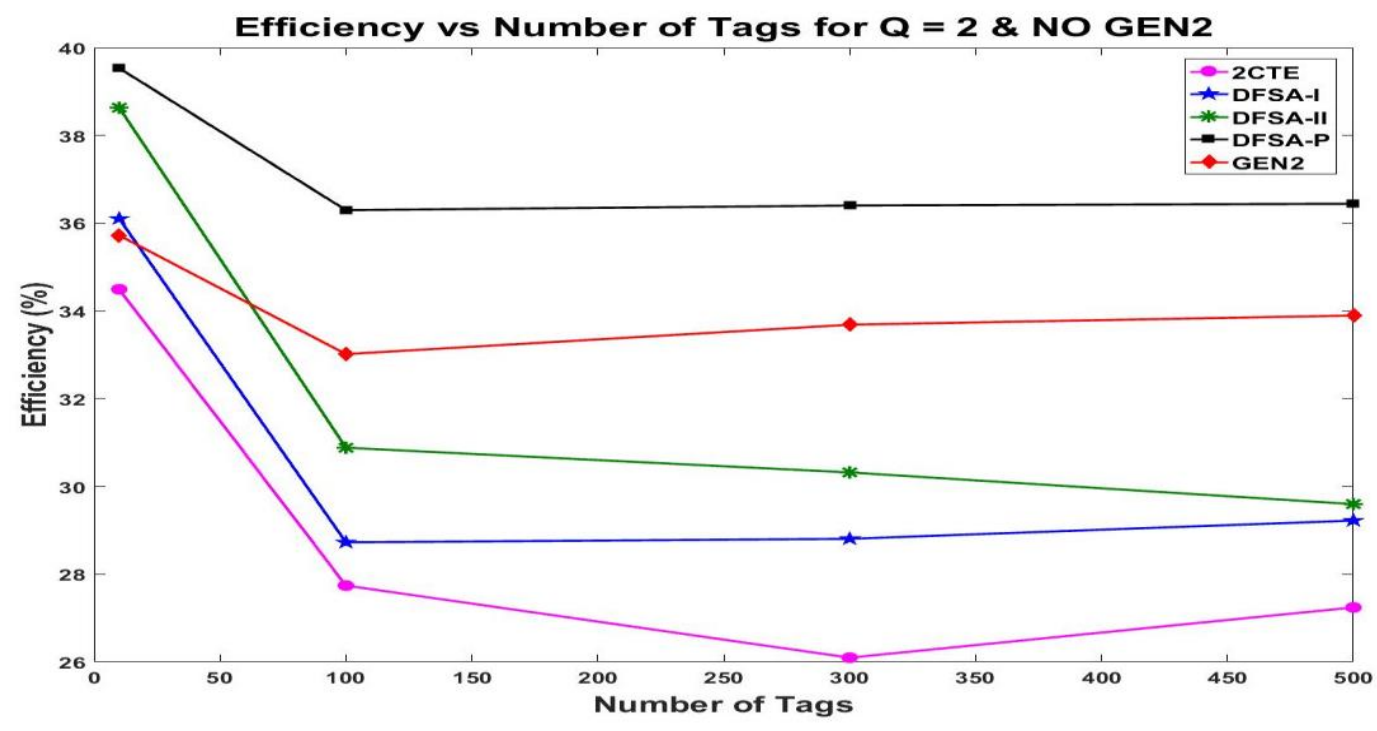

Figure 4.6: Efficiency comparison of the algorithms for $\mathrm{Q}=2$ \& No Gen-2 




Figure 4.7: Efficiency comparison of the algorithms for $\mathrm{Q}=4$ \& No Gen-2

These plots in Figure 4.6 and 4.7 tell a different story. We can see when the algorithms fully replace Gen-2, for tag density up to 50, only DFSA-II can out perform Gen-2, but after approximately 50 tags, Gen-2 takes over.

A more precise and numerical comparison of performances in terms of efficiency and standard deviation can also be seen in tables $4.1-4.6$.

Table 4.1: Performance comparison in terms of efficiency and standard deviation of the algorithms for $\mathrm{Q}=2, \mathrm{C}=0.2 \&$ Frame Size

\begin{tabular}{|c|c|c|c|c|c|c|c|c|c|c|}
\hline \multirow{2}{*}{$\begin{array}{c}\text { No. } \\
\text { of } \\
\text { Tags }\end{array}$} & \multicolumn{2}{|c|}{ DFSA - I } & \multicolumn{2}{c|}{ DFSA - II } & \multicolumn{2}{c|}{ 2CTE } & \multicolumn{2}{|c|}{ Gen-2 } & \multicolumn{2}{c|}{ DFSA - P } \\
\cline { 2 - 10 } & $\begin{array}{c}\text { Eff. } \\
\text { (\%) }\end{array}$ & $\begin{array}{c}\text { Std. } \\
\text { Deviatio } \\
\mathbf{n}\end{array}$ & $\begin{array}{c}\text { Eff. } \\
(\%)\end{array}$ & $\begin{array}{c}\text { Std. } \\
\text { Deviatio } \\
\mathbf{n}\end{array}$ & $\mathbf{E f f . ~ ( \% ) ~}$ & $\begin{array}{c}\text { Std. } \\
\text { Deviatio } \\
\mathbf{n}\end{array}$ & Eff. (\%) & $\begin{array}{c}\text { Std. } \\
\text { Deviatio } \\
\mathbf{n}\end{array}$ & $\begin{array}{c}\text { Eff. } \\
(\%)\end{array}$ & $\begin{array}{c}\text { Std. } \\
\text { Deviatio } \\
\mathbf{n}\end{array}$ \\
\hline 10 & 30.2464 & 15.3759 & $\mathbf{3 8 . 3 6 6 7}$ & 8.7448 & 34.0796 & $\mathbf{7 . 4 3 9 7}$ & 35.7212 & 7.8956 & $\mathbf{3 9 . 3 0 4}$ & 9.3405 \\
\hline 100 & 33.1252 & 2.5238 & $\mathbf{3 3 . 3 1 8 8}$ & 2.5615 & 32.5995 & 2.6695 & 33.0164 & $\mathbf{2 . 4 8 6 9}$ & $\mathbf{3 3 . 3 6 0 5}$ & 2.5407 \\
\hline 300 & 33.7778 & $\mathbf{1 . 4 8 1 1}$ & $\mathbf{3 3 . 8 7 8 1}$ & 1.5797 & 32.6850 & 2.6126 & 33.6865 & 1.5647 & $\mathbf{3 3 . 8 0 9 4}$ & 1.5557 \\
\hline 500 & 33.9804 & 1.2163 & $\mathbf{3 3 . 9 8 8 8}$ & 1.2204 & 32.6869 & 2.5907 & 33.8956 & $\mathbf{1 . 2 1 2 8}$ & $\mathbf{3 4 . 0 2 2 3}$ & 1.2497 \\
\hline
\end{tabular}


Table 4.2: Performance comparison in terms of efficiency and standard deviation of the algorithms for $\mathrm{Q}=4, \mathrm{C}=0.2 \&$ Frame Size

\begin{tabular}{|c|c|c|c|c|c|c|c|c|c|c|}
\hline \multirow{2}{*}{$\begin{array}{c}\text { No. } \\
\text { of } \\
\text { Tags }\end{array}$} & \multicolumn{2}{|c|}{ DFSA - I } & \multicolumn{2}{|c|}{ DFSA - II } & \multicolumn{2}{|c|}{ 2CTE } & \multicolumn{2}{|c|}{ Gen-2 } & \multicolumn{2}{|c|}{ DFSA - P } \\
\hline & $\begin{array}{l}\text { Eff. } \\
(\%)\end{array}$ & $\begin{array}{c}\text { Std. } \\
\text { Deviatio } \\
\mathbf{n}\end{array}$ & $\begin{array}{l}\text { Eff. } \\
(\%)\end{array}$ & $\begin{array}{c}\text { Std. } \\
\text { Deviatio } \\
\mathbf{n}\end{array}$ & Eff. (\%) & $\begin{array}{c}\text { Std. } \\
\text { Deviatio } \\
\mathbf{n}\end{array}$ & Eff. (\%) & $\begin{array}{c}\text { Std. } \\
\text { Deviatio } \\
\mathbf{n}\end{array}$ & Eff. (\%) & $\begin{array}{c}\text { Std. } \\
\text { Deviatio } \\
\text { n }\end{array}$ \\
\hline 10 & 38.1730 & 8.7903 & 39.9726 & 8.9726 & 34.7896 & 8.6706 & 36.6938 & 7.9406 & 39.9449 & 8.8312 \\
\hline 100 & 34.2563 & 2.6965 & 34.4629 & 2.7346 & 33.1234 & 2.7610 & 34.1734 & 2.6747 & 34.4965 & 2.7442 \\
\hline 300 & 34.1483 & 1.6121 & 34.2371 & 1.5791 & 32.8719 & 2.6889 & 34.1311 & 1.5522 & 34.3157 & 1.5697 \\
\hline 500 & 34.0851 & 1.2077 & 34.1806 & 1.2299 & 32.8883 & 2.6757 & 34.1741 & 1.2124 & 34.2009 & 1.2560 \\
\hline
\end{tabular}

Table 4.3: Performance comparison in terms of efficiency and standard deviation of the algorithms for $\mathrm{Q}=2, \mathrm{C}=0.2 \&$ (>Frame Size/2)

\begin{tabular}{|c|c|c|c|c|c|c|c|c|c|c|}
\hline \multirow[b]{2}{*}{$\begin{array}{c}\text { No. } \\
\text { of } \\
\text { Tags }\end{array}$} & \multicolumn{2}{|c|}{ DFSA - I } & \multicolumn{2}{|c|}{ DFSA - II } & \multicolumn{2}{|c|}{ 2CTE } & \multicolumn{2}{|c|}{ Gen-2 } & \multicolumn{2}{|c|}{ DFSA - P } \\
\hline & $\begin{array}{l}\text { Eff. } \\
(\%)\end{array}$ & $\begin{array}{c}\text { Std. } \\
\text { Deviatio } \\
n \\
\end{array}$ & $\begin{array}{l}\text { Eff. } \\
(\%)\end{array}$ & $\begin{array}{c}\text { Std. } \\
\text { Deviatio } \\
n \\
\end{array}$ & Eff. (\%) & $\begin{array}{c}\text { Std. } \\
\text { Deviatio } \\
\text { n } \\
\end{array}$ & Eff. (\%) & $\begin{array}{c}\text { Std. } \\
\text { Deviatio } \\
n\end{array}$ & Eff. (\%) & $\begin{array}{c}\text { Std. } \\
\text { Deviatio } \\
n\end{array}$ \\
\hline 10 & 36.7559 & 7.9856 & 38.4107 & 8.8200 & 34.1229 & 7.1362 & 35.7212 & 7.8956 & 39.7791 & 9.2725 \\
\hline 100 & 33.2267 & 2.5935 & 32.8701 & 2.5966 & 32.3529 & 2.7122 & 33.0164 & 2.4869 & 35.3021 & 2.8387 \\
\hline 300 & 33.7192 & 1.5015 & 33.6628 & 1.6022 & 32.6542 & 2.6023 & 33.6865 & 1.5647 & 34.8442 & 1.6742 \\
\hline 500 & 33.9041 & 1.2016 & 33.8096 & 1.2199 & 32.6685 & 2.5833 & 33.8956 & 1.2128 & 34.5931 & 1.2401 \\
\hline
\end{tabular}

Table 4.4: Performance comparison in terms of efficiency and standard deviation of the algorithms for $\mathrm{Q}=4, \mathrm{C}=0.2 \&(>$ Frame Size/2)

\begin{tabular}{|c|c|c|c|c|c|c|c|c|c|c|}
\hline \multirow{2}{*}{$\begin{array}{c}\text { No. } \\
\text { of } \\
\text { Tags }\end{array}$} & \multicolumn{2}{|c|}{ DFSA - I } & \multicolumn{2}{|c|}{ DFSA - II } & \multicolumn{2}{|c|}{ 2CTE } & \multicolumn{2}{|c|}{ Gen-2 } & \multicolumn{2}{|c|}{ DFSA - P } \\
\hline & $\begin{array}{l}\text { Eff. } \\
(\%)\end{array}$ & $\begin{array}{c}\text { Std. } \\
\text { Deviatio } \\
\mathbf{n}\end{array}$ & $\begin{array}{l}\text { Eff. } \\
(\%)\end{array}$ & $\begin{array}{c}\text { Std. } \\
\text { Deviatio } \\
\text { n }\end{array}$ & Eff. (\%) & $\begin{array}{c}\text { Std. } \\
\text { Deviatio } \\
\text { n }\end{array}$ & Eff. (\%) & $\begin{array}{c}\text { Std. } \\
\text { Deviatio } \\
\mathbf{n}\end{array}$ & $\begin{array}{l}\text { Eff. } \\
(\%)\end{array}$ & $\begin{array}{c}\text { Std. } \\
\text { Deviatio } \\
\text { n }\end{array}$ \\
\hline 10 & 38.7468 & 9.2075 & 39.8472 & 9.4322 & 34.9160 & 8.7949 & 36.6938 & 7.9406 & 41.1367 & 9.8671 \\
\hline 100 & 34.1754 & 2.7046 & 33.8281 & 2.7320 & 32.7911 & 2.7636 & 34.1734 & 2.6747 & 34.6779 & 2.7446 \\
\hline 300 & 34.1306 & 1.6106 & 33.9611 & 1.5882 & 32.8461 & 2.6851 & 34.1311 & 1.5522 & 34.2603 & 1.5966 \\
\hline 500 & 34.0465 & 1.2021 & 34.0735 & 1.2322 & 32.8695 & 2.6722 & 34.1741 & 1.2124 & 34.1717 & 1.2155 \\
\hline
\end{tabular}


Table 4.5: Performance comparison in terms of efficiency and standard deviation of the algorithms for $\mathrm{Q}=2, \mathrm{C}=0.2 \&$ No Gen-2

\begin{tabular}{|c|c|c|c|c|c|c|c|c|c|c|}
\hline \multirow[b]{2}{*}{$\begin{array}{c}\text { No. } \\
\text { of } \\
\text { Tags }\end{array}$} & \multicolumn{2}{|c|}{ DFSA - I } & \multicolumn{2}{|c|}{ DFSA - II } & \multicolumn{2}{|c|}{ 2CTE } & \multicolumn{2}{|c|}{ Gen-2 } & \multicolumn{2}{|c|}{ DFSA - P } \\
\hline & $\begin{array}{l}\text { Eff. } \\
(\%)\end{array}$ & $\begin{array}{c}\text { Std. } \\
\text { Deviatio } \\
\mathbf{n}\end{array}$ & $\begin{array}{l}\text { Eff. } \\
(\%)\end{array}$ & $\begin{array}{c}\text { Std. } \\
\text { Deviatio } \\
\mathbf{n}\end{array}$ & Eff. (\%) & $\begin{array}{c}\text { Std. } \\
\text { Deviatio } \\
\mathbf{n}\end{array}$ & Eff. (\%) & $\begin{array}{c}\text { Std. } \\
\text { Deviatio } \\
\mathbf{n}\end{array}$ & $\begin{array}{l}\text { Eff. } \\
(\%)\end{array}$ & $\begin{array}{c}\text { Std. } \\
\text { Deviatio } \\
\mathbf{n}\end{array}$ \\
\hline 10 & 36.1029 & 8.2952 & 38.6336 & 8.5950 & 34.4941 & 6.6283 & 35.7212 & 7.8956 & 39.5341 & 9.3056 \\
\hline 100 & 28.7261 & 2.8581 & 30.8829 & 2.0561 & 27.7408 & 2.5903 & 33.0164 & 2.4869 & 36.2928 & 2.7255 \\
\hline 300 & 28.8061 & 1.7309 & 30.3195 & 1.2475 & 26.0973 & 1.6970 & 33.6865 & 1.5647 & 36.4028 & 1.5004 \\
\hline 500 & 29.2216 & 1.0073 & 29.5971 & 0.8953 & 27.2420 & 1.9182 & 33.8956 & 1.2128 & 36.4437 & 1.2533 \\
\hline
\end{tabular}

Table 4.6: Performance comparison in terms of efficiency and standard deviation of the algorithms for $\mathrm{Q}=4, \mathrm{C}=0.2 \&$ No Gen-2

\begin{tabular}{|c|c|c|c|c|c|c|c|c|c|c|}
\hline \multirow{2}{*}{$\begin{array}{c}\text { No. } \\
\text { of } \\
\text { Tags }\end{array}$} & \multicolumn{2}{|c|}{ DFSA - I } & \multicolumn{2}{c|}{ DFSA - II } & \multicolumn{2}{c|}{ 2CTE } & \multicolumn{2}{c|}{ Gen-2 } & \multicolumn{2}{c|}{ DFSA - P } \\
\cline { 2 - 10 } & $\begin{array}{c}\text { Eff. } \\
\text { Deviatio } \\
\mathbf{n}\end{array}$ & Eff. (\%) & $\begin{array}{c}\text { Std. } \\
\text { Deviati } \\
\text { on }\end{array}$ & Eff. (\%) & $\begin{array}{c}\text { Std. } \\
\text { Deviatio } \\
\mathbf{n}\end{array}$ & Eff. (\%) & $\begin{array}{c}\text { Std. } \\
\text { Deviatio } \\
\mathbf{n}\end{array}$ & $\begin{array}{c}\text { Eff. } \\
(\%)\end{array}$ & $\begin{array}{c}\text { Std. } \\
\text { Deviatio } \\
\mathbf{n}\end{array}$ \\
\hline 10 & 34.8616 & 8.1240 & $\mathbf{4 0 . 1 6 1 8}$ & 9.0587 & 33.8109 & 8.6592 & 36.6938 & $\mathbf{7 . 9 4 0 6}$ & $\mathbf{4 0 . 2 4 1 9}$ & 9.0816 \\
\hline 100 & 29.8044 & 3.0294 & 32.1351 & $\mathbf{2 . 2 0 1 6}$ & 28.6736 & 2.7754 & $\mathbf{3 4 . 1 7 3 4}$ & 2.6747 & $\mathbf{3 4 . 8 5 5 2}$ & 2.4978 \\
\hline 300 & 29.1125 & 1.7153 & 30.6931 & $\mathbf{1 . 2 6 6 8}$ & 26.4974 & 1.7527 & $\mathbf{3 4 . 1 3 1 1}$ & 1.5522 & $\mathbf{3 5 . 8 4 1 9}$ & 1.5309 \\
\hline 500 & 29.4303 & 1.0089 & 29.8559 & $\mathbf{0 . 9 0 1 1}$ & 27.3303 & 1.9914 & $\mathbf{3 4 . 1 7 4 1}$ & 1.2124 & $\mathbf{3 6 . 1 7 3 8}$ & 1.2245 \\
\hline
\end{tabular}

If we look at the performances of the algorithms as represented both in Fig. $4.2-4.5 \&$ Table 4.1 - 4.4, when incorporated with EPC Gen-2 for Q $=2 \& 4$ and for different scenarios, one can see that for low tag density $(\mathrm{N}=10$ - 100) environments, DFSA-I and II have higher system efficiency than pure EPC Gen-2 (with DFSA-II having slightly better performance), where 2CTE method lags. However, as one proceeds towards higher tag density environments we can see that DFSA-I, DFSA-II and pure EPC Gen-2 show almost identical performances. The slight differences in efficiencies for $\mathrm{Q}=4$ and >frame size/2 (Fig. $4.5 \&$ Table 4.4) case are not statistically significant. In all cases, although 2CTE method shows lesser performance than other techniques, 
it is noticeable that it gives a consistent result independent of the scenario and choice of Q value as described by the authors of the original 2CTE method article [6]. When algorithms are used without Gen-2 (Figure 4.6 - $4.7 \&$ Table $4.5-4.6$ ), we can see a similar situation where for a low number of tags $(\mathrm{N}=10-50)$, DFSA-II provides better performance than pure EPC Gen- 2, and DFSA-I perform equally well with 2CTE method lagging behind. However, for higher tag density environments pure EPC Gen-2 has consistently and significantly higher efficiency than all other algorithms, whereas DFSA-P shows the highest performance irrespective of the scenario and Q values as expected [1] [4]. We can also see from Table $4.1-4.6$ that the algorithms behaved quite similarly in terms of standard deviation on efficiency. For all the cases, the standard deviation is highest for low tag densities (especially $\mathrm{N}=10$ ) and as the number of tags goes higher, the deviation on efficiency stabilizes as expected from any probabilistic algorithm.

\subsubsection{Effects of Utilization Rate and Estimation Error on Efficiency}

As mentioned earlier, in this study, we will analyze the effects of these parameters on DFSA-I, DFSA-II and DFSA-P and learn the behavior of these algorithms. We plotted the estimation error and the utilization rate of the algorithms against efficiency to see what kind of effect these parameters have on the overall efficiency of each algorithm. We evaluated these phenomena for a specific number of tag population (for $\mathrm{N}=100$ ) to have a more in-depth look. For this we first plotted the histogram of the utilization rate and estimation error to see the distribution of data points. Then we plotted utilization rate and estimation error for these data points and their corresponding efficiencies.

\subsubsection{Utilization Rate vs Efficiency}

For analyzing the effect of utilization rate on efficiency, first we study the histogram of the utilization rate to see the distribution of data points. 
We plotted the effect of utilization rate on the efficiency for two scenarios:

i) When the frame update occurs at the end of the frame, and

ii) When the frame update occurs after mid-frame.

We do not consider the no Gen-2 case as the utilization rate is obviously $100 \%$. We observed that regardless of the type of algorithm the rate of utilization is very low around $5 \%$ and $10 \%$ for the first and second scenarios respectively. This may be because of the fact that EPC gen2 cuts frames short more often than not.

The following figure shows the histograms of the utilization rate for DFSA-I, DFSA-II and DFSA-P respectively for $Q=2$ and for the case where frame update occurs at the end of the frame.


Figure 4.8: Histogram of utilization rate for DFSA-I, II \& $\mathrm{P}(\mathrm{Q}=2, \mathrm{C}=0.2 \&$ Frame Size $)$ 


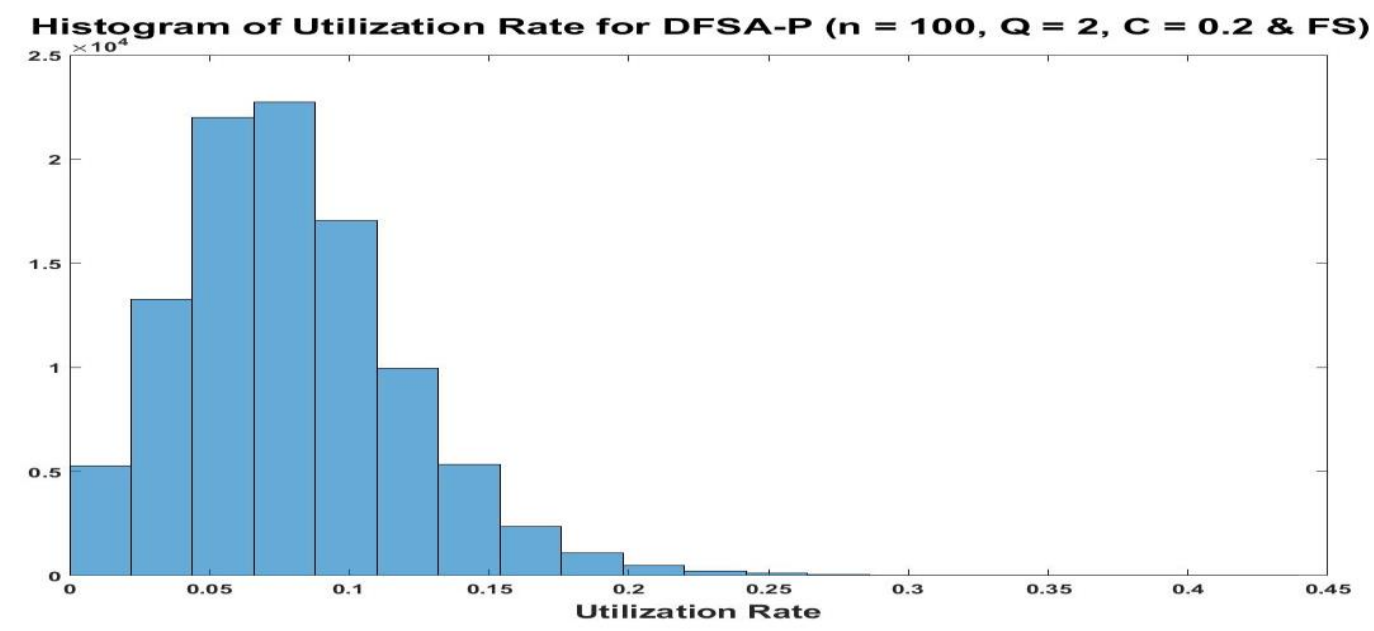

Figure 4.8 (Continued)

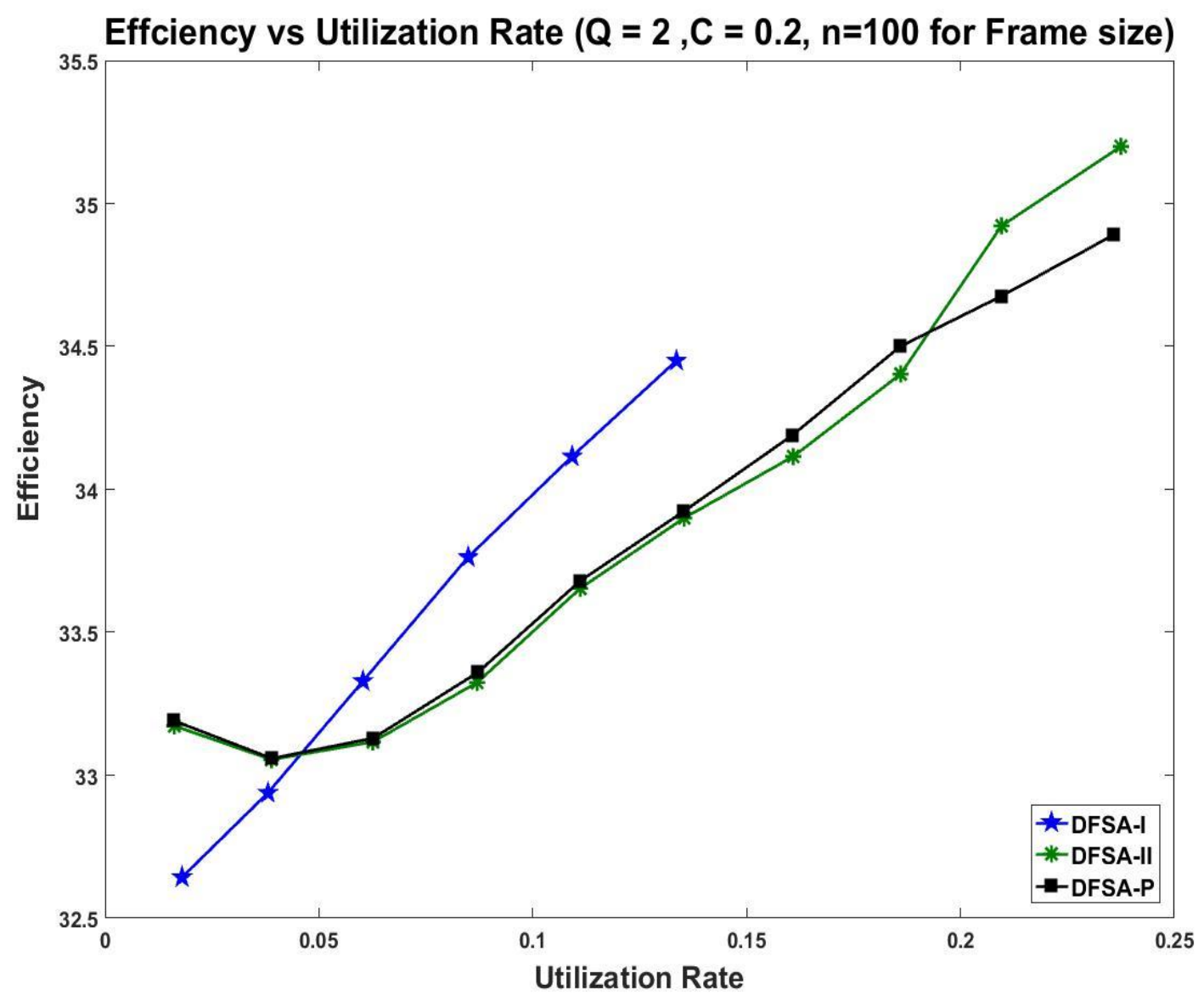

Figure 4.9: Utilization rate vs efficiency for DFSA-I, II \& $\mathrm{P}(\mathrm{Q}=2, \mathrm{C}=0.2 \&$ Frame Size $)$ 
We can see from Figure. 4.8, that for each algorithm the utilization rate ranges from $0 \%$ to $10 \%$ and majority of the data points accumulate around 5\% which is low. However, from Fig. 4.9 we can see an upward trend in efficiency with increasing utilization rate regardless of the type of algorithms which is very promising. DFSA-I demonstrates the steepest increase compared to the other two algorithms. On the other hand, DFSA-II and DFSA-P show quite similar performance.

The next figure is the histograms of the utilization rate for DFSA-I, DFSA-II and DFSA-P respectively for $\mathrm{Q}=4$ and for the case where frame update occurs at the end of the frame.


Figure 4.10: Histogram of utilization rate for DFSA-I, II \& P $(\mathrm{Q}=4, \mathrm{C}=0.2 \&$ Frame Size $)$ 




Figure 4.10 (Continued)

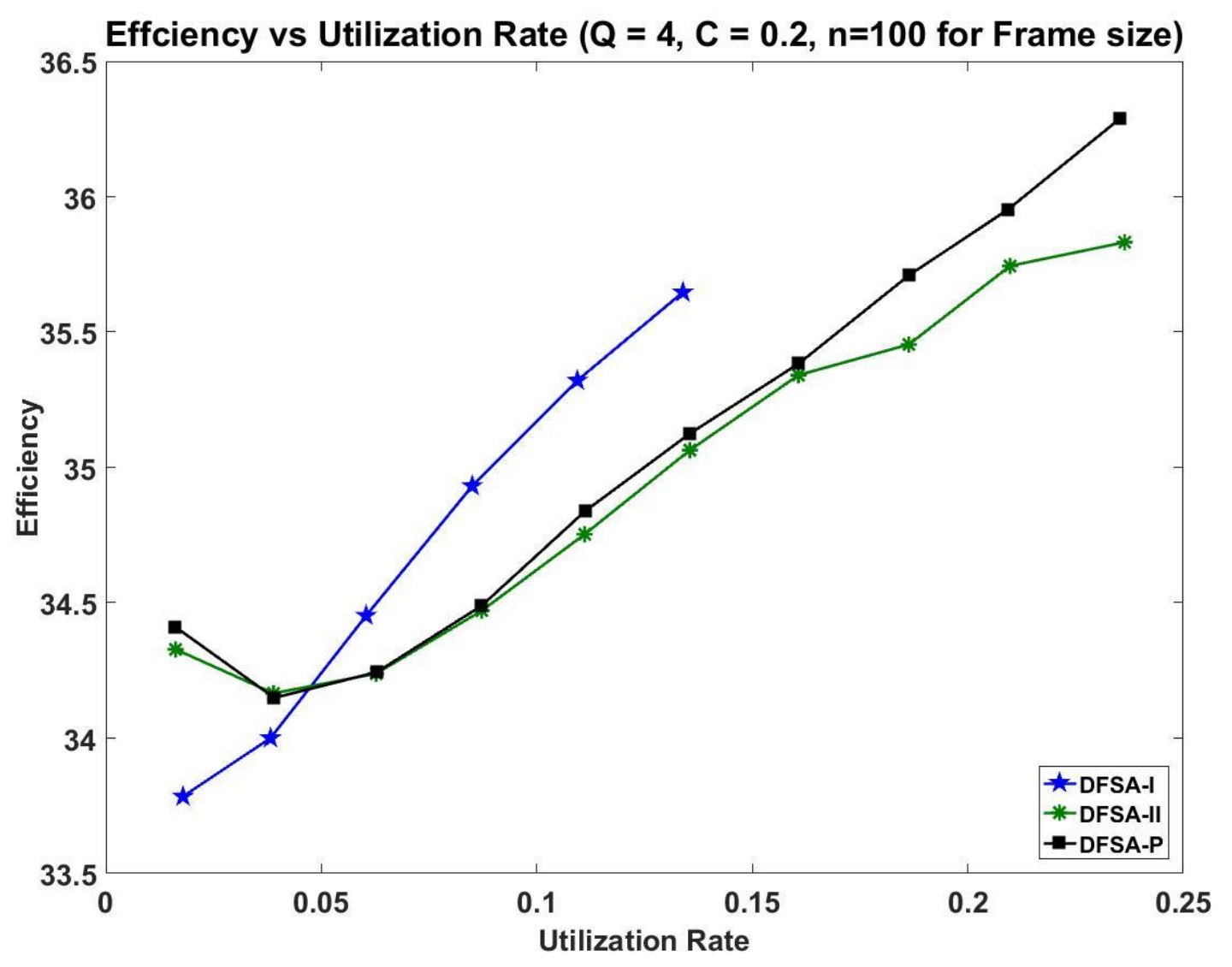

Figure 4.11: Utilization rate vs efficiency for DFSA-I, II \& P ( $Q=4, C=0.2 \&$ Frame Size $)$ 
In Figure 4.10 we can see a similar kind of distribution as the previous one, which depicts low utilization rate. Figure 4.11 also shows the same kind of upward trend for all the algorithms. DFSA-I has the highest slope among the three algorithms for this set of parameters as well.

Rest of the figures in this section deals with the scenario where the frame update occurs after mid-frame. The first figure for the second scenario shows the histograms of the utilization rates for DFSA-I, DFSA-II and DFSA-P respectively for Q = 2 and for the case where frame update occurs after mid-frame.
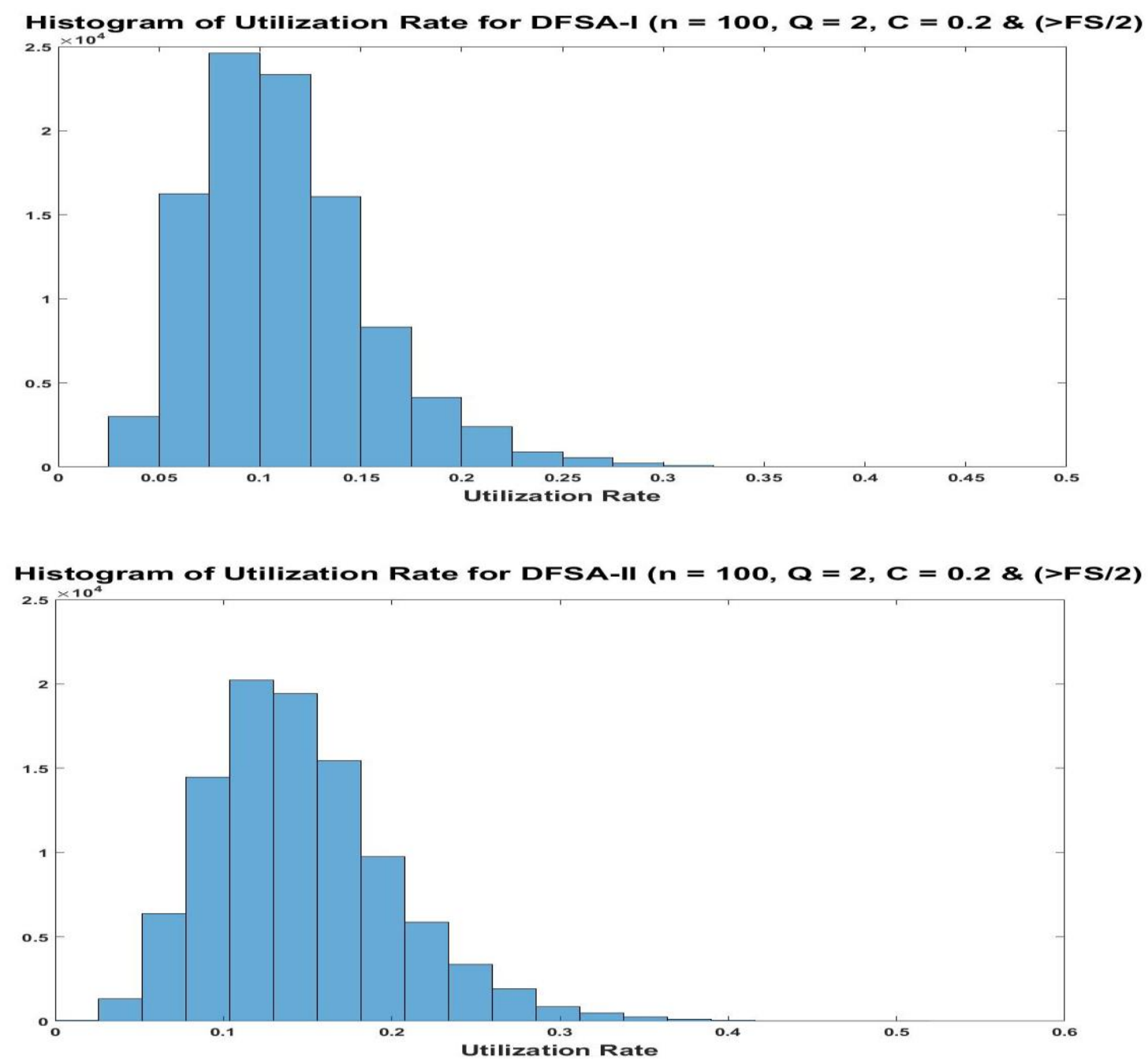

Figure 4.12: Histogram of utilization rate for DFSA-I, II \& $\mathrm{P}(\mathrm{Q}=2, \mathrm{C}=0.2 \&>$ Frame Size/2) 


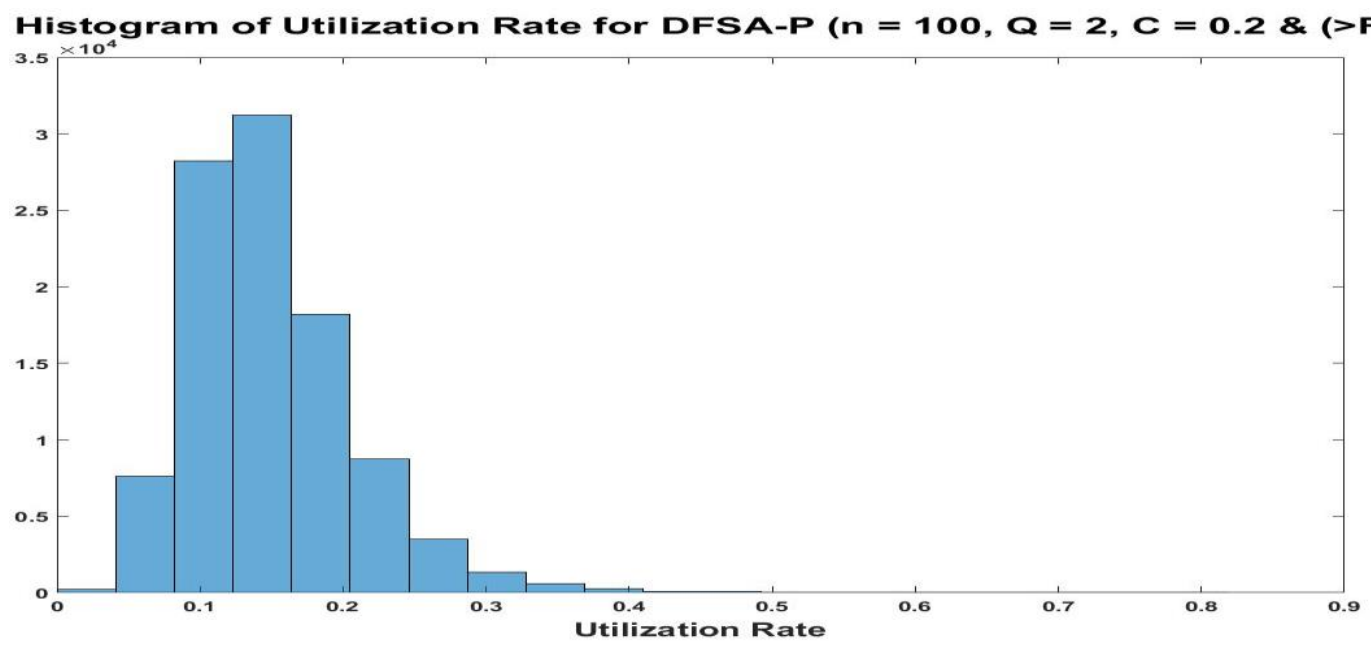

Figure 4.12 (Continued)

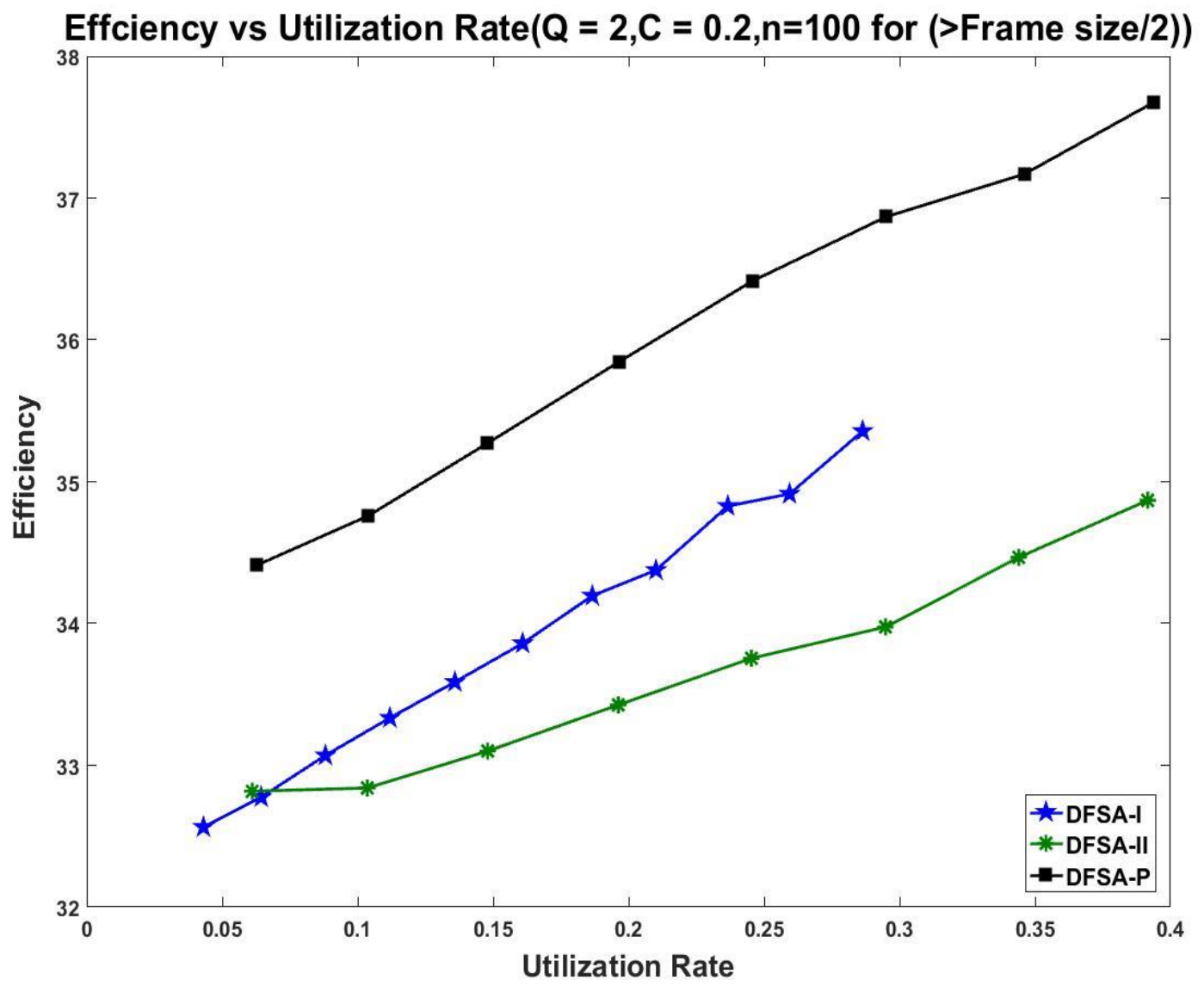

Figure 4.13: Utilization rate vs efficiency for DFSA-I, II \& $\mathrm{P}(\mathrm{Q}=2, \mathrm{C}=0.2 \&>$ Frame Size/2) 
Figure 4.12 shows the distribution of all three algorithms for $Q=2$ and when the frame update occurs after mid frame. As expected, we can see from the distribution that for $>$ frame Size/2 case, the rate is higher than the first scenario where the frame update occurs at the end of the frame as it is more probable that a cycle will reach mid-frame. However, in Figure 4.13 the trend is still the same but the slope drops for all the algorithms compared to the previously mentioned scenario. Also, the slope is kind of similar for all the algorithms except for DFSA-II being just a bit lower which can be overlooked as the variation is not statistically significant.

The figure below shows the histograms of the utilization rate for DFSA-I, DFSA-II and DFSA-P respectively for $\mathrm{Q}=4$ and for the case where frame update occurs after mid-frame.
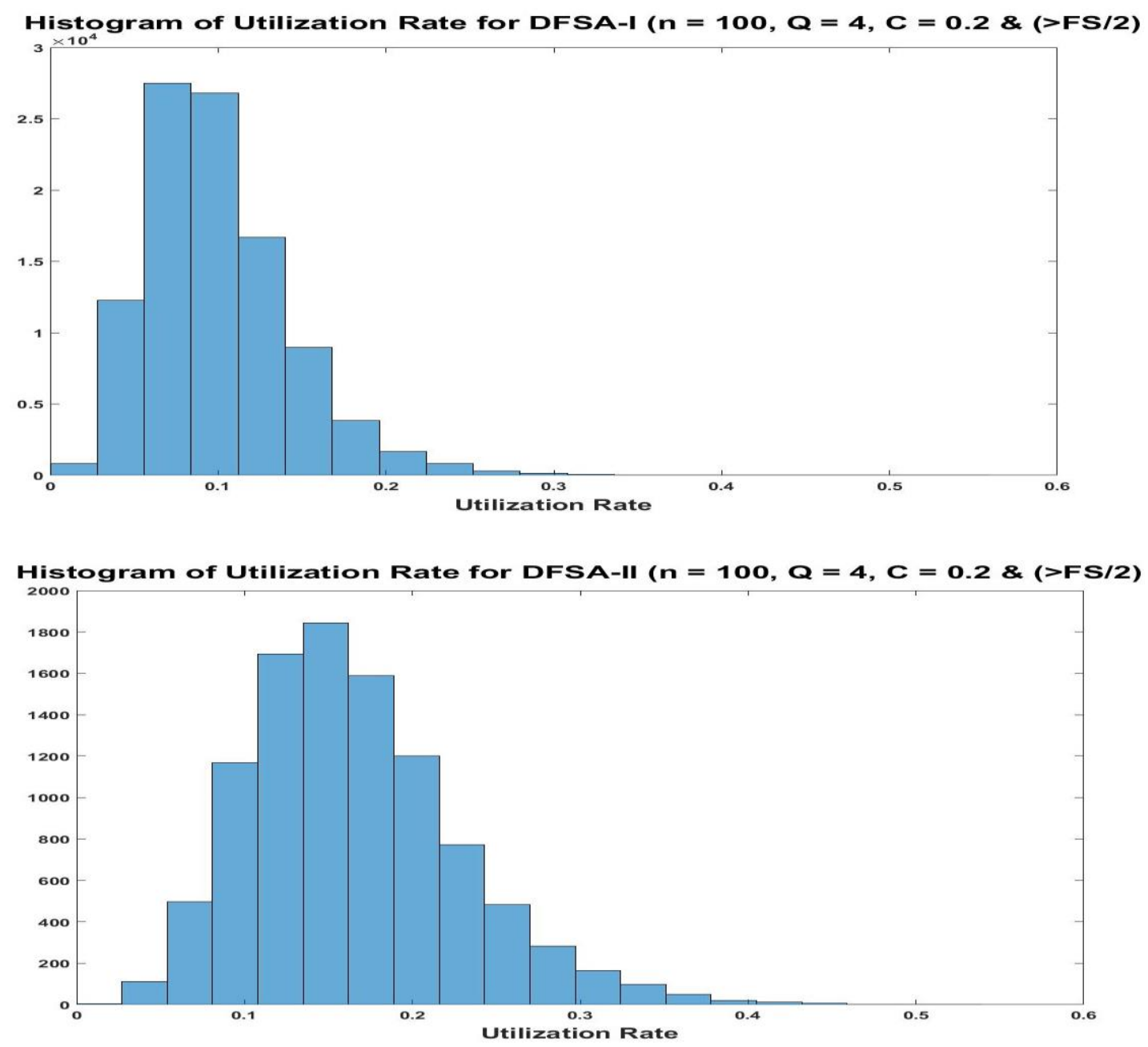

Figure 4.14: Histogram of utilization rate for DFSA-I, II \& $\mathrm{P}(\mathrm{Q}=4, \mathrm{C}=0.2 \&>$ Frame Size/2) 




Figure 4.14 (Continued)

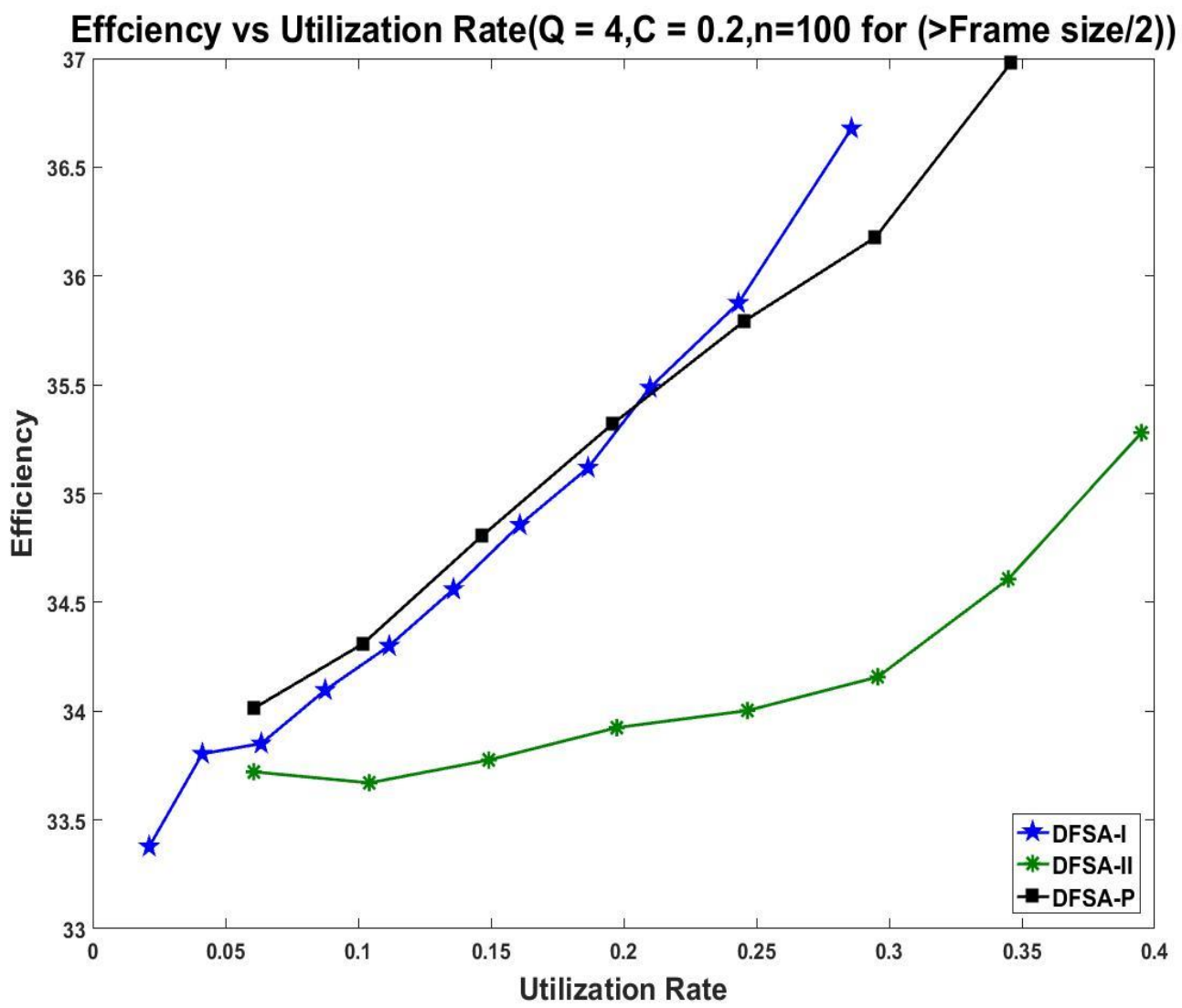

Figure 4.15: Utilization rate vs efficiency for DFSA-I, II \& $\mathrm{P}(\mathrm{Q}=4, \mathrm{C}=0.2 \&>$ Frame Size/2)

From Figure 4.14 we can see a similar kind of distribution for the utilization rate, where most of the data points are gathered around $10 \%$ to $20 \%$ which is $5 \%$ to $15 \%$ higher than that of 
the first scenario as expected. Figure 4.15 shows a similar trend but DFSA-I and DFSA-P shows higher slope compared to the $\mathrm{Q}=2$ case for the same scenario, whereas DFSA-II is more flat.

For all the conditions and parameters, we considered for analyzing the effect of utilization rate on efficiency for the chosen algorithms, we can summarize by saying that even though the algorithms weren't utilized often, increasing utilization rates have yielded higher efficiencies. Hence, if these algorithms can be better utilized, the efficiency can further be improved.

\subsubsection{Estimation Error vs Efficiency}

We analyzed the relationship between the estimation error and channel efficiency. No Gen2 case was used to isolate the effect of prediction accuracy. In other cases, the utilization rates were very low and Gen-2 dictates over $90 \%$ - $95 \%$ of the time preventing the algorithms to exhibit their true performance in estimating the number of tags.

We plotted the efficiency against the estimation error separately for each algorithm. The following two plots are for DFSA-I algorithm for $\mathrm{Q}=2 \& 4$.
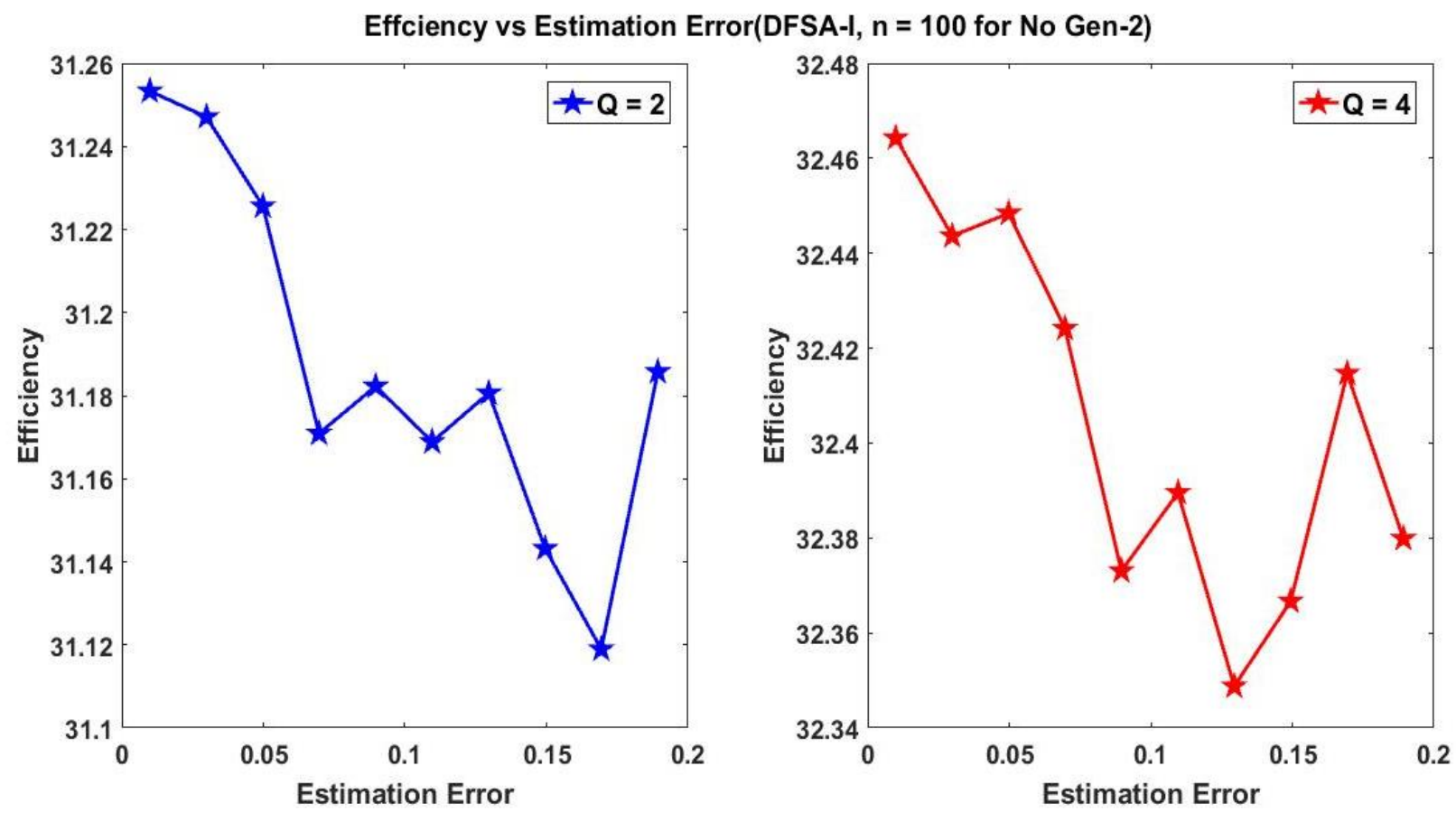

Figure 4.16: Estimation error vs efficiency for DFSA-I $(\mathrm{Q}=2 \& 4, \mathrm{C}=0.2 \&$ No Gen-2) 
We can see from these plots that the algorithm behaves similarly for both $Q=2 \& 4$. One can observe an overall downward trend, as expected, although there is some fluctuation and the efficiency differences between the minimum and maximum points of the graphs are insignificant. To further study this behavior we plotted the histogram of the estimation error for DFSA-I to have a clear vision on how many times the number of tags were under and overestimated.
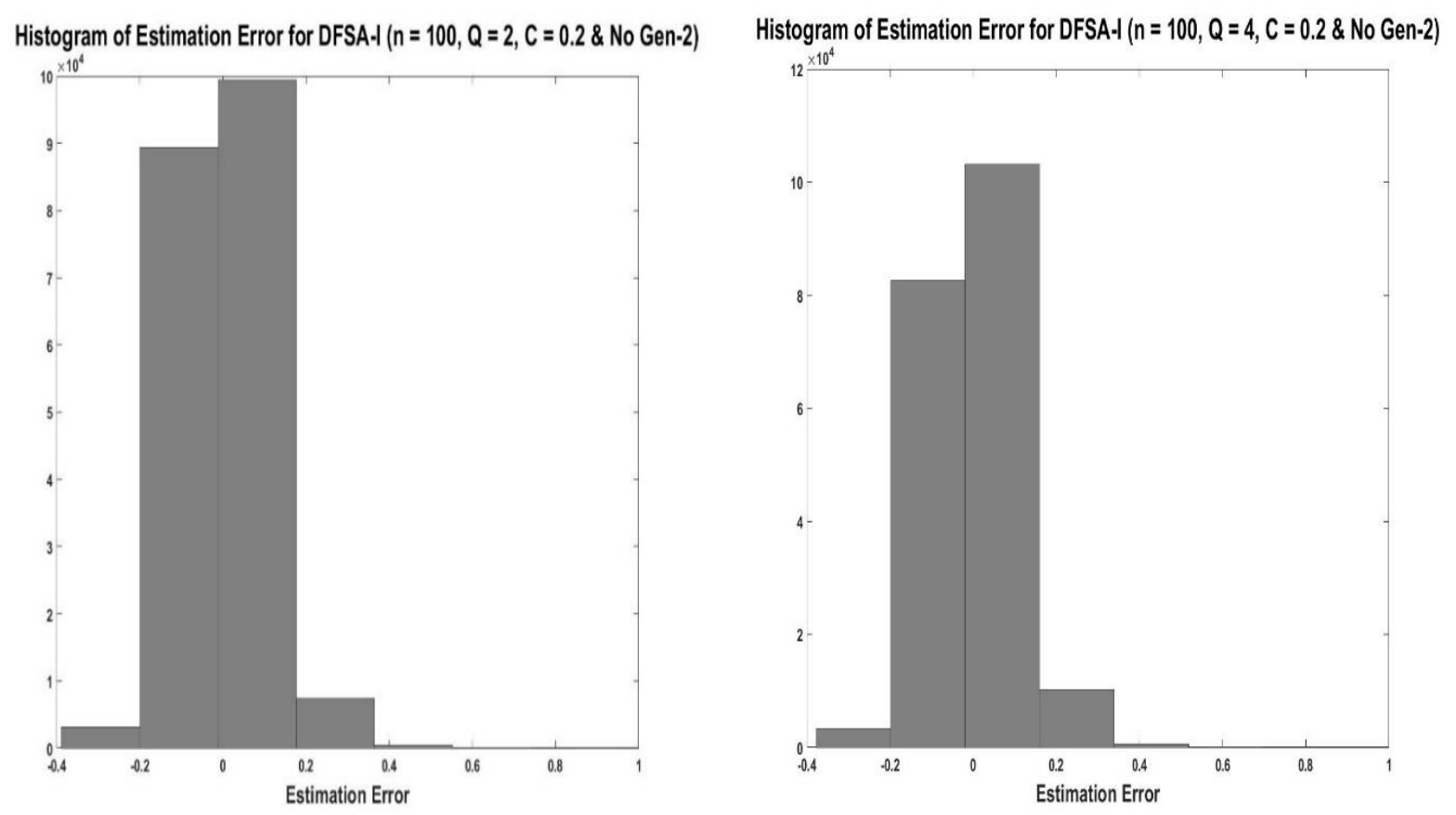

Figure 4.17: Histograms of estimation error for DFSA-I $(Q=2 \& 4, C=0.2 \&$ No Gen-2)

We can see from Figure 4.17 that the estimation error is roughly equally distributed around zero mean which can explain the fluctuations in Figure 4.16. One can assume that, if the estimation error is equally and normally distributed around zero, it has no statistically significant impact on the efficiency as the over and underestimations have a cancelling effect on one another.

On the other hand, Figure 4.18 shows the estimation error versus efficiency curves for the same settings for DFSA-II algorithm. Here, we can see that as the estimation error gets higher efficiency increases, which is against the common logic. To study this behavior closer, we plotted the histogram of the estimation error for DFSA-II. 



Figure 4.18: Estimation error vs efficiency for DFSA-II ( $Q=2 \& 4, \mathrm{C}=0.2 \&$ No Gen-2)

Histogram of Estimation Error for DFSA-II $(n=100, Q=2, C=0.2 \&$ No Gen-2)

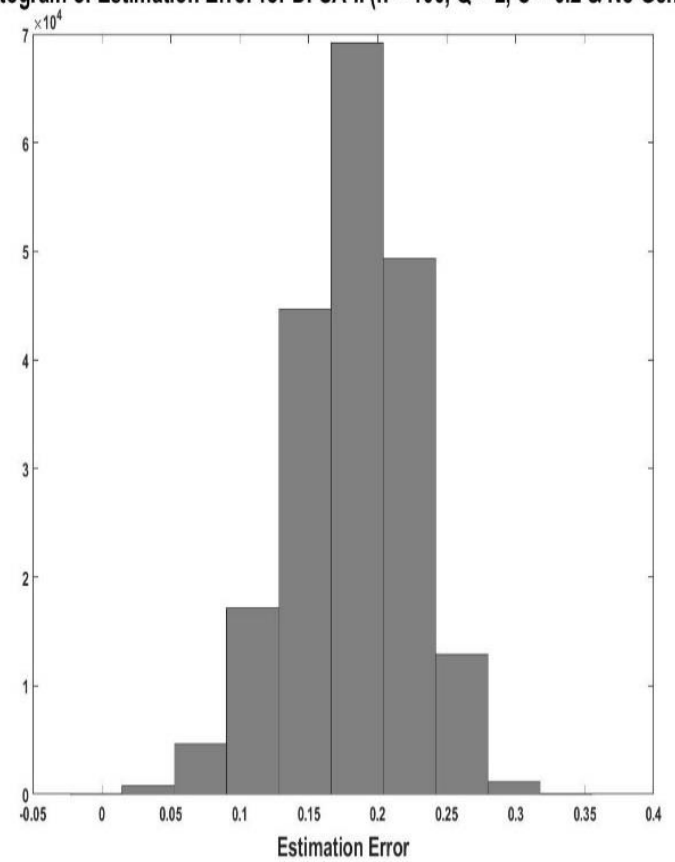

Histogram of Estimation Error for DFSA-II $(n=100, Q=4, C=0.2 \&$ No Gen-2)



Figure 4.19: Histogram of estimation error for DFSA-II ( $Q=2 \& 4, \mathrm{C}=0.2 \&$ No Gen-2) 
Figure 4.19 shows that the estimation error for this case has a different statistical distribution with non-zero mean. In other words, this algorithm is more inclined to underestimate the number of tags than overestimate. Considering the fact that the inclination of this algorithm towards underestimation is somehow helping the system performance, we designed a new experiment. We modified DFSA-P and intentionally introduced biased error with a similar distribution to DFSA-II. The histogram of the estimation error for DFSA-P and Q $=4$ is as follows.

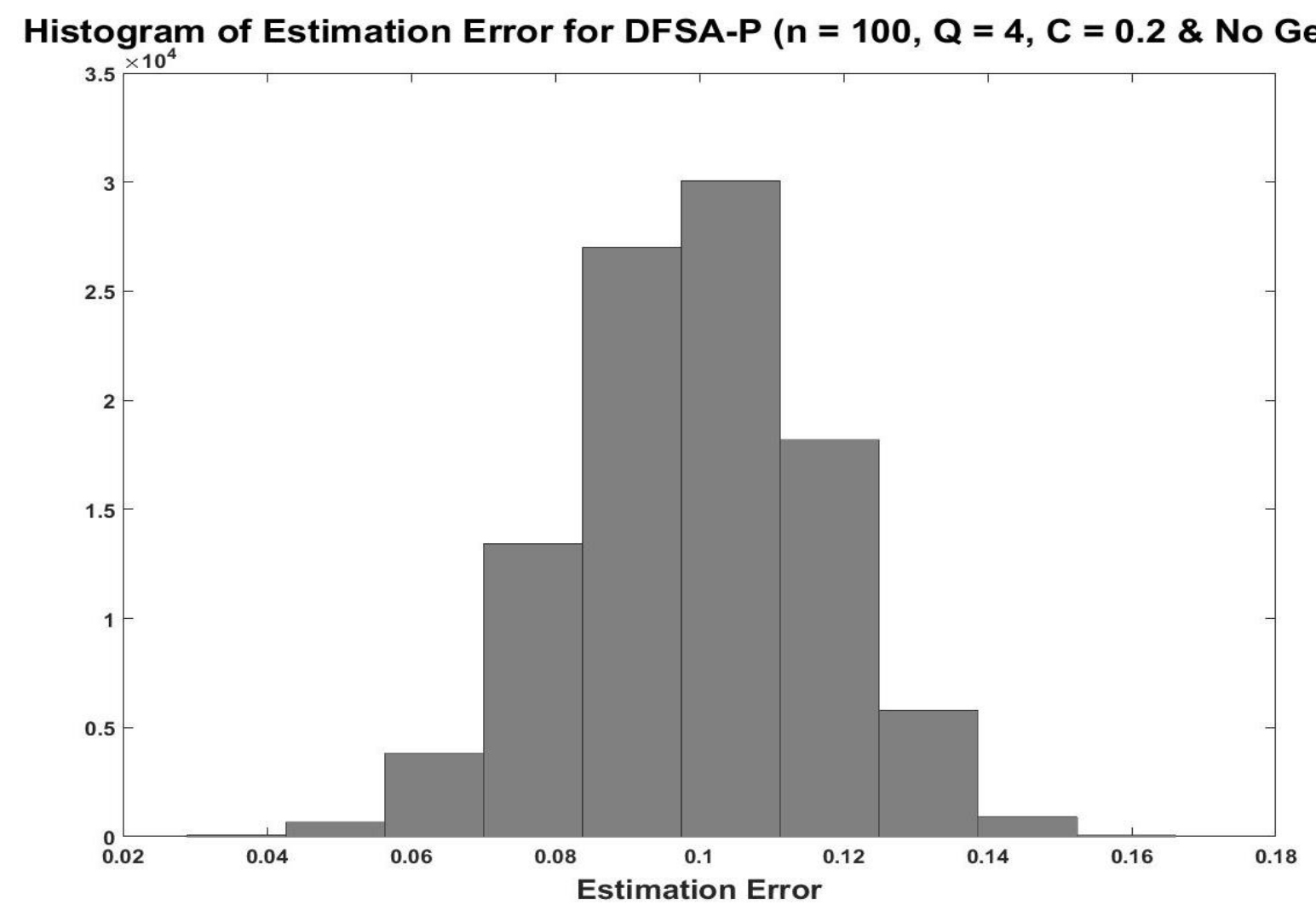

Figure 4.20: Histogram of estimation error for DFSA-P $(Q=4, C=0.2 \&$ No Gen-2)

As we can see from Figure 4.20 error-modified DFSA-P now underestimates the number of tags just like DFSA-II. However, when we plotted estimation error against efficiency to compare with DFSA-II we obtained Figure 4.21 which is similar to DFSA-I (decreasing efficiency with increasing estimation error). 


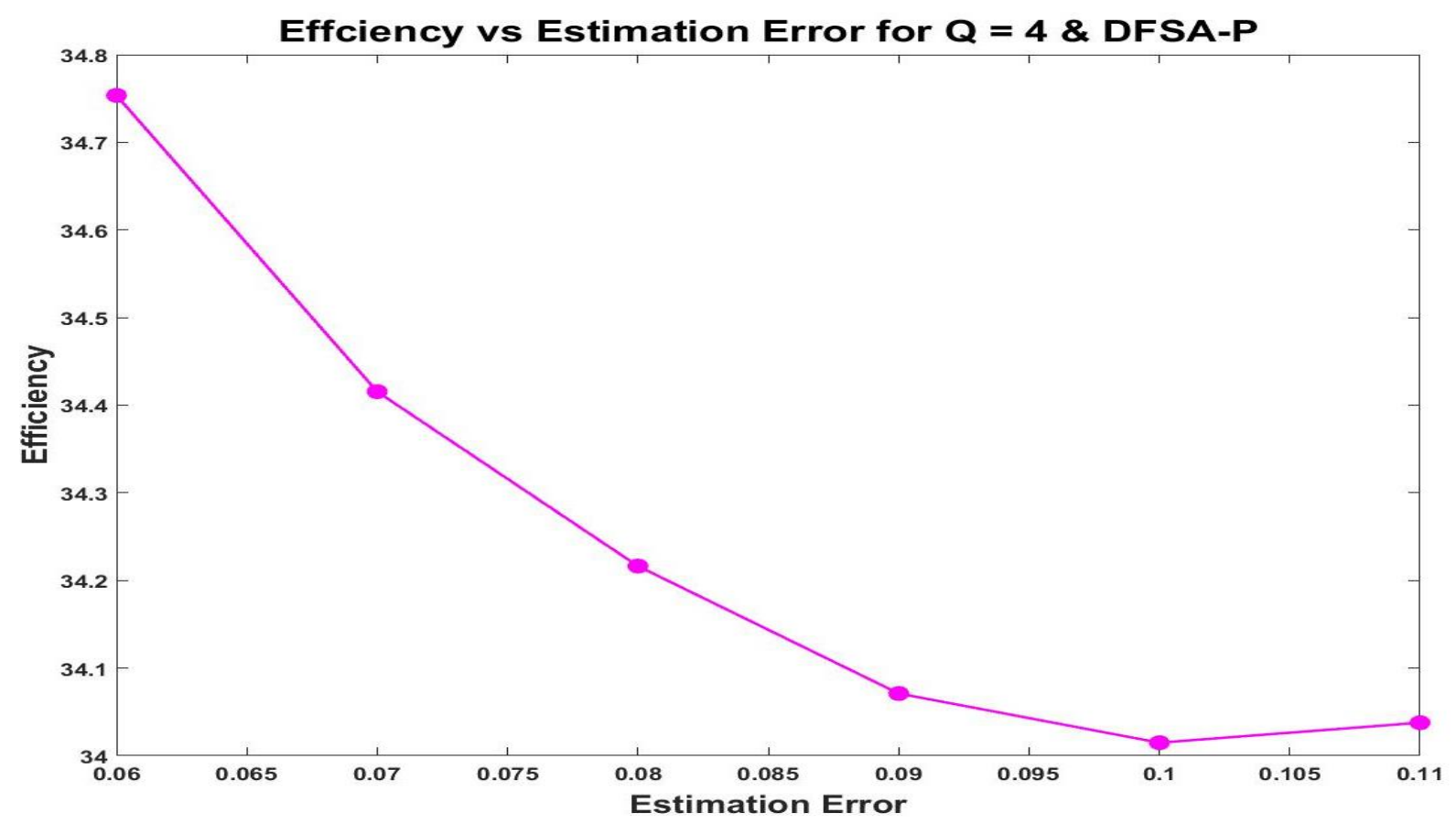

Figure 4.21: Estimation error vs efficiency for DFSA-P $(Q=4, C=0.2 \&$ No Gen-2)

From Figure 4.21, it is apparent that underestimating the number of tags does not necessarily help the system efficiency. In this case, the unique behavior of DFSA-II needs to be studied further as part of future work. 


\section{CHAPTER 5: CONCLUSION}

In this study, we present a novel framework for true assessment and comprehensive analysis of probabilistic tag estimation algorithms when integrated with RFID Gen-2 in terms of statistically significant correlations between channel efficiency, algorithm estimation accuracy and utilization rate.

As far as the channel efficiency is concerned, the results clearly demonstrate that at low tag density environments two of the tested algorithms may improve the efficiency of pure EPC Gen-2 frame allocation scheme. However, as the number of tags increases, simulating high-volume application scenarios, none of the algorithms actually presented a statistically significant increase to efficiency regardless of how they are incorporated with EPC Gen-2 unless the estimation was perfectly accurate as in the case of DFSA-P. We conclude that unless one alters the existing hardware infrastructure including the RFID readers and tags across a multitude of industries, which would cost billions of dollars, EPC Gen-2 performs respectably in approaching the theoretical maximum efficiency allowed by randomized frame-slotted ALOHA algorithms.

We also performed an in-depth analysis of the two probabilistic algorithms, DFSA-I and DFSA-II as these algorithms show highest system efficiency among the chosen ones. In general, the channel efficiency seems to increase with increasing utilization rate (especially for DFSA-I algorithm). A different procedure of utilizing the algorithm more, may boost the efficiency higher. However, this increase is bounded as $100 \%$ utilization rate (no Gen-2) results in worse performance than $0 \%$ utilization rate (Pure Gen-2). As far as the estimation error is concerned, 
when it is equally and normally distributed around zero (0), there is no statistically significant impact on channel efficiency which we can see in the case of DFSA-I. On the other hand, if the error distribution has a non-zero mean, it may have an opposite impact, which needs to be studied further as part of the future work. In designing the experimental setup, we used a scalable and flexible simulation framework and created a light-weight, verifiable Gen-2 simulation tool to measure these performance parameters as it is very difficult, if not impossible, to calculate system performance analytically. We hope that other researchers can easily use this framework to test and compare more algorithms in the literature with Gen-2 and other DFSA based counterparts. 


\section{REFERENCES}

[1] Roberti, M., Wal-Mart begins RFID process changes. RFID Journal, 2005: p. 1385-

[2] Ilie-Zudor, E., et al., The RFID technology and its current applications. proceedings of the modern information technology in the innovation processes of the industrial enterprisesMITIP, 2006. 5(7).

[3] Bolic, M., D. Simplot-Ryl, and I. Stojmenovic, RFID systems: research trends and challenges. 2010: John Wiley \& Sons.

[4] EPCglobal, E., Radio-Frequency Identity Protocols Generation-2 UHF RFID specification for RFID air interface protocol for communications at $860 \mathrm{MHz}-960 \mathrm{MHz}$, version 2.0. 0 . GS1, EPCglobal, 2013.

[5] Uysal, I. and N. Khanna. Q-frame-collision-counter: A novel and dynamic approach to RFID Gen 2's Q algorithm. in RFID Technology and Applications (RFID-TA), 2015 IEEE International Conference on. 2015. IEEE.

[6] Klair, D.K., K.-W. Chin, and R. Raad, A survey and tutorial of RFID anti-collision protocols. IEEE Communications Surveys \& Tutorials, 2010. 12(3): p. 400-421.

[7] Finkenzeller, K., RFID handbook: fundamentals and applications in contactless smart cards, radio frequency identification and near-field communication. 2010: John Wiley \& Sons.

[8] Shields, A., et al., Radio Frequency Identification (RFID). Wiley Encyclopedia of Electrical and Electronics Engineering.

[9] Want, R., An introduction to RFID technology. IEEE pervasive computing, 2006. 5(1): p. 25-33.

[10] Lee, S.-R., S.-D. Joo, and C.-W. Lee. An enhanced dynamic framed slotted ALOHA algorithm for RFID tag identification. in Mobile and Ubiquitous Systems: Networking and Services, 2005. MobiQuitous 2005. The Second Annual International Conference on. 2005. IEEE.

[11] Kodialam, M. and T. Nandagopal. Fast and reliable estimation schemes in RFID systems. in Proceedings of the 12th annual international conference on Mobile computing and networking. 2006. ACM. 
[12] Patil, K., et al., Comparative Analysis of Anti-Collision Protocols in RFID. IJAEMS: Open Access International Journal: Infogain Publication. 2(Issue-4).

[13] Hush, D.R. and C. Wood. Analysis of tree algorithms for RFID arbitration. in Information Theory, 1998. Proceedings. 1998 IEEE International Symposium on. 1998. IEEE.

[14] Capetanakis, J., Tree algorithms for packet broadcast channels. IEEE transactions on information theory, 1979. 25(5): p. 505-515.

[15] Abramson, N. THE ALOHA SYSTEM: another alternative for computer communications. in Proceedings of the November 17-19, 1970, fall joint computer conference. 1970. ACM.

[16] Wong, C. and Q. Feng, Grouping based bit-slot ALOHA protocol for tag anti-collision in RFID systems. IEEE Communications Letters, 2007. 11(12).

[17] Yihong, C., et al., Multiple-bits-slot reservation ALOHA protocol for tag identification. IEEE Transactions on Consumer Electronics, 2013. 59(1): p. 93-100.

[18] Su, J., et al., An effective frame breaking policy for dynamic framed slotted ALOHA in RFID. IEEE Communications Letters, 2016. 20(4): p. 692-695.

[19] Liu, X., et al., Multi-category RFID estimation. IEEE/ACM transactions on networking, 2017. 25(1): p. 264-277.

[20] Šolić, P., J. Radić, and N. Rožić, Energy efficient tag estimation method for ALOHA-based RFID systems. IEEE Sensors Journal, 2014. 14(10): p. 3637-3647.

[21] Kong, L., et al. A parallel identification protocol for RFID systems. in INFOCOM, 2014 Proceedings IEEE. 2014. IEEE.

[22] Arjona, L., et al., Fast fuzzy anti-collision protocol for the RFID standard EPC Gen-2. Electronics Letters, 2016. 52(8): p. 663-664.

[23] Chen, W.-T., Optimal Frame Length Analysis and an Efficient Anti-Collision Algorithm With Early Adjustment of Frame Length for RFID Systems. IEEE Transactions on Vehicular Technology, 2016. 65(5): p. 3342-3348.

[24] Chen, W.-T. A new RFID anti-collision algorithm for the EPCglobal UHF class-1 generation-2 standard. in Ubiquitous Intelligence \& Computing and 9th International Conference on Autonomic \& Trusted Computing (UIC/ATC), 2012 9th International Conference on. 2012. IEEE.

[25] Vales-Alonso, J., et al., Multiframe maximum-likelihood tag estimation for RFID anticollision protocols. IEEE Transactions on Industrial Informatics, 2011. 7(3): p. 487496. 
[26] Chen, W.-T., A feasible and easy-to-implement anticollision algorithm for the EPCglobal UHF class-1 generation-2 RFID protocol. IEEE Transactions on Automation Science and Engineering, 2014. 11(2): p. 485-491.

[27] Cha, J.-R. and J.-H. Kim. Novel anti-collision algorithms for fast object identification in RFID system. in Parallel and Distributed Systems, 2005. Proceedings. 11th International Conference on. 2005. IEEE.

[28] HajMirzaei, M. and Z. Adelani. 2 Conditional tag estimation method for DFSA algorithms in RFID systems. in Computer and Knowledge Engineering (ICCKE), 2014 4th International eConference on. 2014. IEEE.

[29] Zhang, X., T. Chen, and F. Hu, RFID Tags Estimation through the Statistics Method for ALOHA. International Journal of Computer and Electrical Engineering, 2014. 6(3): p. 259.

[30] Landaluce, H., A. Perallos, and I. Angulo. A simulation tool for RFID EPC Gen2 protocol. in Information Systems and Technologies (CISTI), 2012 7th Iberian Conference on. 2012. IEEE. 